\title{
On a Multi-criterion Problem of Planning Maritime Cargo Transportation
}

\author{
Ruslans Aleksejevs ${ }^{1}$, Raufs Guseinovs ${ }^{2}$, Alexander N. Medvedev ${ }^{3}$ and Sharif E. Guseynov ${ }^{4}$ \\ 1. Riga State Gymnasium No. 1, 8 Raina Boulevard, Riga LV-1050, Latvia; \\ 2. Faculty of Humanities, Leiden University, Rapenburg 70, 2311 EZ Leiden, the Netherlands; \\ 3. Department of Aviation Transport, Transport and Telecommunication Institute, 1 Lomonosov Street, Riga LV-1019, Latvia; \\ 4. Institute of Fundamental Science and Innovative Technologies, Liepaja University, 14 Liela Street, Liepaja LV-3401, Latvia
}

\begin{abstract}
In this work, we consider a specific problem of optimal planning of maritime transportation of multiproduct cargo by ships of one (so-called "corporate strategy") or several (so-called "partially corporate strategy") companies: the core of the problem consists of the existence of the network of intermediate seaports (i.e., transitional seaports), where for every ship arrived the cargo handling is done, and which are situated between the starting and the finishing seaports. In this work, there are mathematical models built from scratch in the form of multi-criterion optimization problem; according to the properties of the criteria and structure of the feasible solution set; are formulated different optimality conditions; are analysed different approaches for finding effective solutions (i.e., Pareto optimal solutions) and for check of the given solutions' effectiveness. In addition, in this work, there is considered and analysed well-known method of contraction of the Pareto boundary (goal attainment method of Gembicki), then, it is used for reducing the built models to a one-criterion problem of linear programming.
\end{abstract}

Key words: Marine cargo transportation, mathematical modeling, multi-criterion optimization, ill-posedness.

\section{Introduction}

Maritime cargo transportation is a complicated multistage transportation-and-manufacturing process, in what, besides sender, carrier and recipient of cargo, other natural and legal persons do also participate [1]: agents and forwarders; banks and insurance companies; seaport workers and workers of logistic complexes of intermodal and multimodal transportation systems; representatives of state supervisory authorities; etc. All these transportation-and-manufacturing process participants at different stages of cargo transportation enter into specific legal relationships among themselves for solving particular problems, which assists with the achievement of the common objective - punctual (fastness), inexpensive (economic expedience) and secure (safety and stability) delivery

Corresponding author: Alexander N. Medvedev, professor; research fields: transport and logistics systems, materials-handling system, and software development. E-mail: Medvedevs.A@tsi.lv. of the cargo to the destination. For instance, Brukhis and Luschan [2] consider various optimization models solving the fleet size decision making problems involving fleet changes during several planning periods. In the article, the choice of the best suited model structure of deterministic nature is described. The author of this article shows that decisions made are sufficiently sensitive to small changes of the input parameters, and introduces so-called "scenario algorithm" for treating the input parameters for the purpose of minimizing losses coming from likely errors, which are a consequence of the aforesaid sensitivity. Further, James and Kendall [3] suggest a model integrating supply, production networks and sustainable freight transportation for strategic and tactical decision making. The objective function considers sourcing, production and transportation costs as well as carbon dioxide emissions as environmental impacts of transport over a multimodal network. The work [4] in detail expounds a metaheuristic algorithm 
based on a genetic algorithm. The purpose of the developed algorithm consists in solving the problem of car carriers work's efficiency as well as in creating a maritime transportation planning support system, thus making it possible to prepare various alternatives, evaluate them and, consequently, support user's decision making. On other important problems related to functioning of logistic system, can be found in the readable and deservedly popular textbooks $[5,6]$.

In the beginning of $21 \mathrm{st}$ century, the world production entered a qualitatively new phase of technological changes, because of which afterwards, the structure of international trade was changed itself, which led to radical reconstruction of all schemes of its transport maintenance, particularly, occurred fundamental changes in the structure of cargo base: in the international trade, the portion of extractive and primary sectors (agriculture, fishery, hunt and forestry) is permanently falling. For the last 20 years, the portion of transportation expenses in the international price of a good has fallen, on average, $10 \%$, but the cost of the transported goods has spiked up, which led to increase of insurance premiums: in 2014, in comparison with 2000, they increased 1.2 times (in comparison with 1980 - 7 times), and according to predictions, the growth trend will be stable at least until 2025. That is why in these conditions qualitative factors of transportation service (speed; cost; safety and stability; service), which define the level of competitive ability of national transport systems, in particular, the level of maritime cargo transportation, are becoming priority-driven. Some EU countries like the Baltic States (Latvia, Lithuania, and Estonia), the Netherlands, Germany, Hungary, Czech Republic, and Poland, owning small territories, use their advantageous geographical locations to turn transit into significant income items for their national budgets. For instance, the Netherlands is the most important transit crossing of $\mathrm{EU}$, and the portion of incomes from transit of freight flows going through the Port of Rotterdam forms more than $45 \%$ of total volume of export of services of the Netherlands [7]: at present, the Port of Rotterdam is the fourth busiest port in the world based on cargo turnover volumes (450 million tons), and it follows Shanghai (650 million tons), Ningbo/Zhoushan (627 million tons), and Singapore (502.5 million tons). In view of the fact than the investigated in this work, particular problem is initiated by one of the features of transit cargo transportation through the seaports of the Baltic States, we will consider the freight flows accomplished through these seaports in details. Maritime cargo is the cornerstone of transport and logistics in the Baltic States. Top 10 ports on the eastern coast of the Baltic Sea have seen a steady growth in total cargo turnover over the last years, accounting for an average increase of 5\%-8\% year-on-year. In terms of cargo transshipment, the ports of the eastern part of the Baltic Sea (the Eastern Baltic) serve mainly the east-west cargo flow corridor with the majority of cargo originating from the CIS (Commonwealth of Independent States) countries. While the three key Russian ports (Ust-Luga, St. Petersburg, and Primorsk) are the obvious leaders in maritime cargo transshipment for the key types of cargo, including oil and coal, the largest ports of the Baltic States still account for a significant share in the total cargo turnover in the region. The cost of reloading is not the key factor defining competitiveness of an individual port, as it is one of the many components in the overall transshipment chain. With respect to the east-west cargo flows, the total cost of transshipment heavily depends on the railway tariffs as well as the distance to the port, and therefore one of the primary determinants of the competitiveness of a port is its geographical location. The total volume of goods flowing through the seaports in the eastern part of the Baltic Sea reached about 370 million tonnes (Fig. 1) in 2014 with $42 \%$ going through the seaports of the Baltic States.

The dynamics of cargo turnover of seaports of Latvia, Lithuania, Estonia and Russia (data on Russia covers only Russian seaports in the Baltic Sea basin) for 


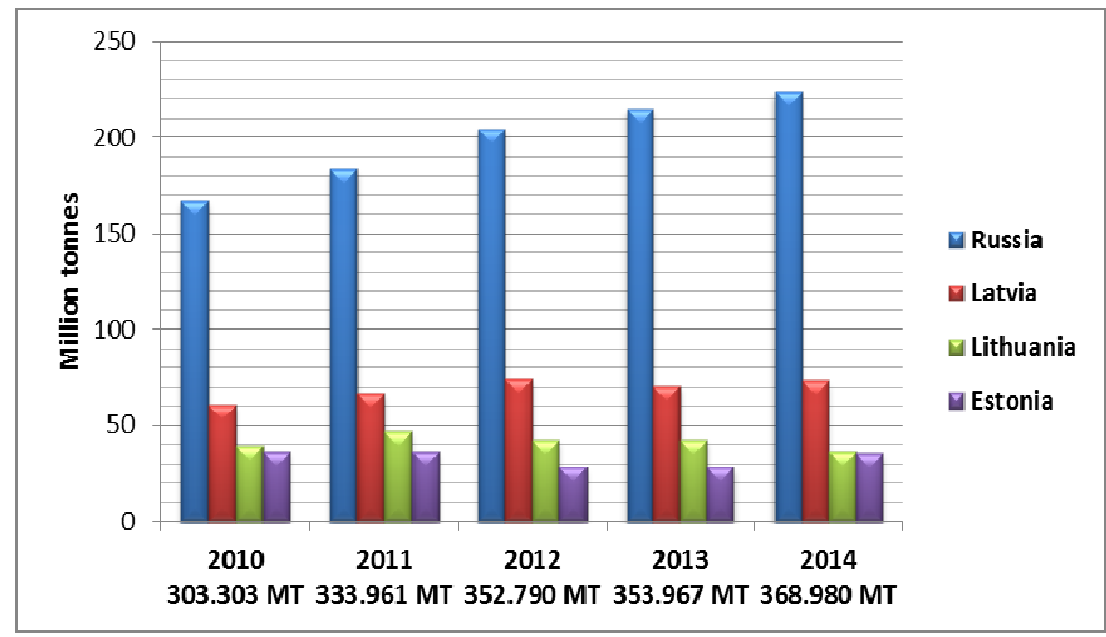

Fig. 1 Cargo turnover in the Eastern Baltic seaports, 2010-20140.

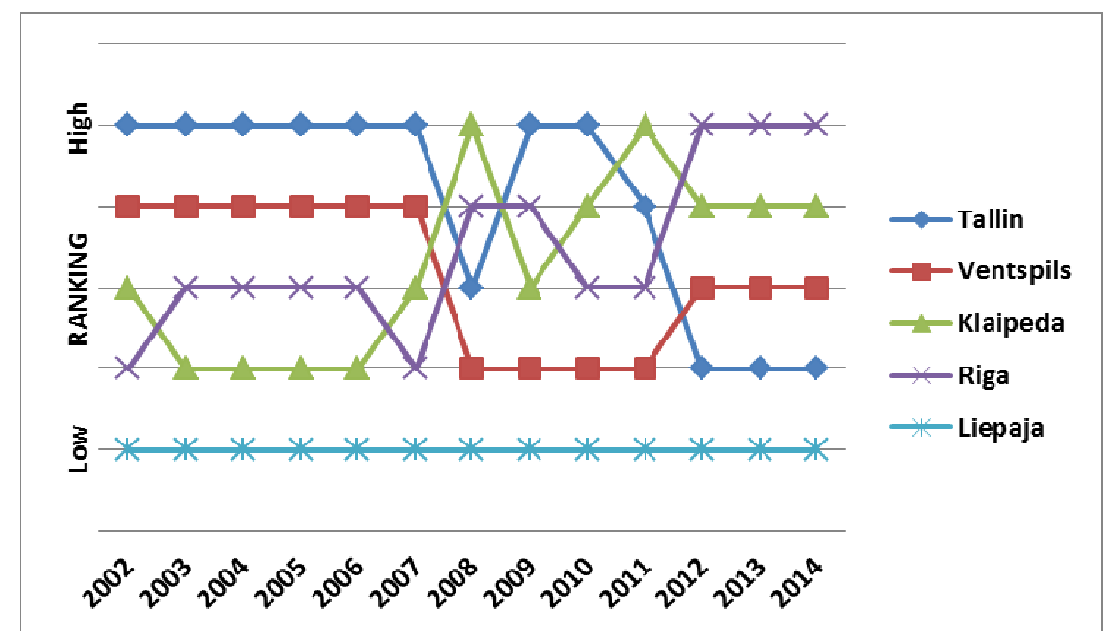

Fig. 2 Ranking of seaports of the Baltic States by total cargo turnover, 2002-2014.

2010-2014 is shown below (Fig. 1). Figs. 2 and 3 show the ranking of seaports of the Baltic States according to total cargo turnover and key technical specifications, respectively [8]. Fig. 4 shows the ranking of top 10 seaports on the eastern coast of the Baltic Sea according to the volumes of cargo flows of eight main cargo types [8]. Although the majority of the Eastern Baltic Sea cargo shipments originate from or head to Russia and other CIS countries, Baltic States altogether still control $40 \%-45 \%$ of cargo flows in the region, while the rest is shipped through Russian seaports. The seaport of Riga, leading among the Baltic States in 2012-2014, ranks 4th in the Eastern Baltic Sea region (Fig. 4), right behind the biggest seaports Primorsk, Ust-Luga and St. Petersburg. While the seaports of the
Baltic States have to make continuous effort to secure their competitive position in the region, the fact that they continuously retain an extensive share of Russian cargo transshipment is a sign of competitive advantage in particular types of cargo, which may be used as the basis for further development. One of the reasons for long-term success of the Baltic States in maritime cargo transportation is partial ownership of seaport terminals by cargo holders, who wish to secure transshipment corridors. This is especially evident with respect to coal and fertilizer terminals, resulting in stable volumes of cargo shipped through such terminals. Additionally, the Baltic States have a favourable logistical position with multiple railway connections to CIS and Asian countries. Regular container 


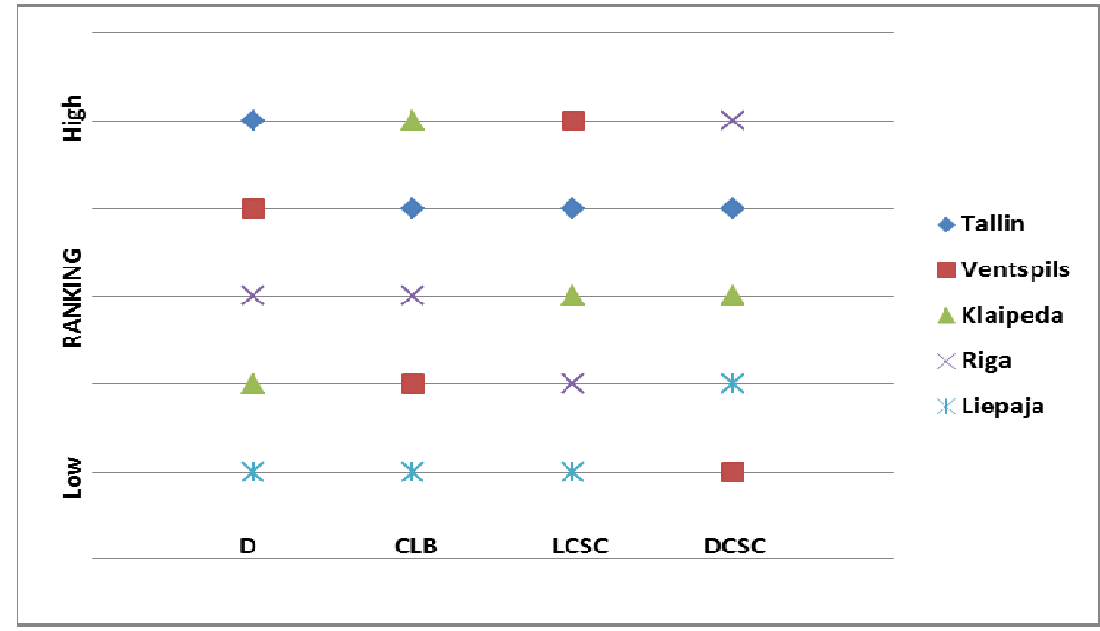

Fig. 3 Ranking of seaports of the Baltic States according to their key technical specifications. On the abscissa, the following notations occur: D—depth; CLB—cumulative length of berths; LCSC—liquid cargo storage capacity; DCSC—dry cargo storage capacity.

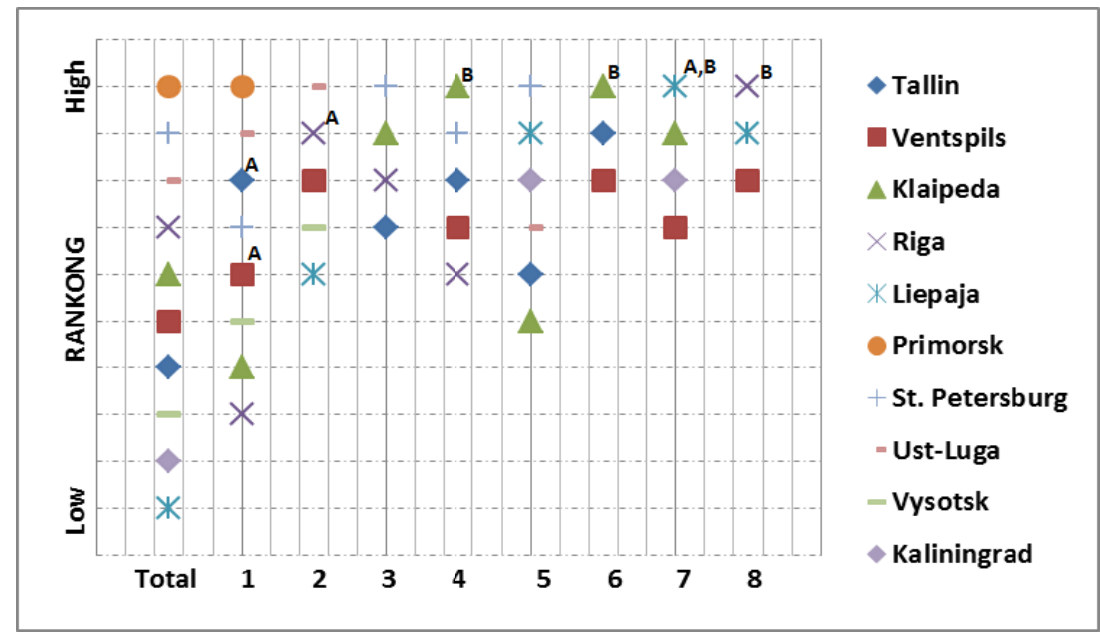

Fig. 4 Ranking of the Eastern Baltic seaports by volumes of cargo flows, 2012-2014. On the abscissa, the following notations occur: total indicates the total cargo turnover; 1 -crude oil, oil and oil products; 2 -coal; 3-containers; 4-fertilizers; 5-metals, metal products and scrap metal; 6-roll-on-roll-off ferry; 7-agricultural.

trains run to/from Russia, Belarus, Ukraine, Kazakhstan, Uzbekistan, Afghanistan and China. Furthermore, special container trains VIKING (Klaipeda $\leftrightarrow$ Black Sea), ZUBR (Estonia $\leftrightarrow$ Latvia $\leftrightarrow$ Black Sea) and BALTIC-TRANSIT (Baltic States $\leftrightarrow$ Central Asia) run several times per week, securing direct access to the continent [9-11]. Nevertheless, a favourable geographic location of seaports (controlled and partially controlled key parameters (hereinafter for conciseness, parameters): goods origin; transportation distance to the seaports; cargo flow corridors; presence of regular shipping lines; proximity of large consumer markets; etc.) is not a competitive advantage per se, as the competitiveness of the seaport is also impacted by other factors including politics and regulations (parameters: open borders; trade limitations; political influence on decisions; tariffs; customs; MARPOL Convention; etc.), governance (parameters: transparency; cooperation with the seaport's authority; certainty of governance decisions; cooperation with railway infrastructure operators; custom duties; etc.), technical specifications (parameters: maximum ship dimensions; ice conditions; quality of the seaport's infrastructure; specified equipment; etc.), service 
(parameters: service equality; reliability; speed, tracking; damage protection; preplanning; additional service offering; etc.), and other key factors that are beyond the control of the terminal or the seaport. Analyzing the available data (received from open as well as from private sources), speaking about the competitive ability of the Baltic States seaports, it is necessary to emphasize the growing tendency of involvement of the highly skilled specialist in all spheres of these seaports activity: the salary is regularly raised and social benefits and entitlements are improved; educational courses are regularly conducted by high-class professors and scientists; promising students higher education in needed spheres is paid under the terms of them working for seaports during a determined period of time after their graduation; etc. That is why one aspect of organization of work in the Baltic States seaports is application of scientific approach (particularly, mathematical modeling and mathematical methods) for solving current problems, as well as for developing of scientifically substantiated policy for medium-term and long-term planning of development of the seaports.

In this work, we investigate a specific problem, which is largely typical exactly for the Baltic States seaports. The key part of the problem consists in the following: it is required to create a plan of maximal multiproduct cargo transportation by every ship through the given sea route such that the transportation expenses are minimal if: (1) the cost of transportation of one unit of cargo between any two intermediate seaports by every ship is minimal; (2) the volumes of cargo unloaded and/or loaded in every intermediate seaport are known; (3) it is known the volume of intended to be transported accumulated cargo in every intermediate seaport; (4) it is known the shipload of every ship after the intended to be transported to this seaport cargo is unloaded from it. It is obvious that in the formulated problem every intermediate seaport is simultaneously a departure and destination point. In the work [12], reasons of this specific cargo transportation are expanded, and is made comparative analysis in comparison with other sea routes in transpacific, transatlantic and Asia $\rightarrow$ Europe directions for whose intermediate seaports this specific character of transportation is not so typical: in the work, cargo transportations performed by ships of three companies "Maersk Line", "Mediterranean Shipping Company S.A." and "CMA CGM" are considered.

In the considered particular problem with respect to the ships performing transportations through the given sea route, we can say the following: the ships can belong to one company, and, therefore, in this case we have a corporate strategy for performing a transportation; all or part of the ships can belong to different companies, and, therefore, in this case we can speak about a partially corporate strategy of performing a transportation. Obviously, in the case of partially corporate strategy, it is possible to add to the formulation of the problem different conditions and constraints and, as result, obtain various problems according to their degree of complexity and purpose.

\section{Construction of Quantitative Model}

The problem described in the introduction, can be schematically presented in the form of the directed graph. Before we begin the model building for the considered problem, let us introduce the following denotations: $n \in \mathbb{N}$ denotes the amount of seaports between which the cargo transportation is performed; $\left[T_{\text {start }}, T_{\text {end }}\right]$ denotes a period of time during which the cargo transportation is performed; $K \in \mathbb{N}$ denotes the total amount of ships used during the period of time $\left[T_{\text {start }}, T_{\text {end }}\right]$ for cargo transportation along the route; $\mathbb{R}_{++}^{m} \stackrel{\text { def }}{\equiv}\left\{y \in \mathbb{R}^{m}: y_{i}>0 \forall i=\overline{1, m}\right\}$ denotes a positive orthant of the space $\mathbb{R}^{m}$, particularly, if $m=1$ we have $\mathbb{R}_{++}^{1} \stackrel{\text { def }}{\equiv}(0,+\infty) ; \quad \mathbb{R}_{+}^{m} \stackrel{\text { def }}{\equiv}\left\{y \in \mathbb{R}^{m}: y_{i} \geq 0 \quad \forall i=\overline{1, m}\right\}$ denotes a nonnegative orthant of the space $\mathbb{R}^{m}$, in particular, if $m=1$, we have $\mathbb{R}_{+}^{1} \stackrel{\text { def }}{\equiv}[0,+\infty)$; 
$c_{i, i+j}^{k}(t):\left[T_{\text {start }}, T_{\text {end }}\right] \rightarrow \mathbb{R}_{++}^{1}$ denotes the cost of transportation of an unit of the cargo loaded in $i$-th $(i=\overline{1,(n-1)})$ seaport which has to be transported to $(i+j)$-ths $(j=\overline{1,(n-i)})$ seaports by the $k$-th $(k=\overline{1, K})$ ship. In this work, we consider only the case, when for $\forall t \in\left[T_{\text {start }}, T_{\text {end }}\right]$ holds $c_{i, i+j}^{k}(t) \equiv c_{i, i+j}^{k}=$ const; $\quad p_{i, i+j}^{k} \in[0,100]$ denotes the percent of the cargo which is being transported by the $k$-th $(k=\overline{1, K})$ ship and which, firstly, was loaded in the $i$-th $(i=\overline{1,(n-1)})$ seaport and, secondly, after its transportation in $(i+j)$-th $(j=\overline{1,(n-i)})$ seaport "settles down"/unloads in this seaport. Obviously, for each fixed $k \in\{1,2, \ldots, K\}$, the identity $\sum_{j=1}^{n-i} p_{i, i+j}^{k}=100$ must hold for each $i=\overline{1,(n-1)} ; \quad a_{i} \in \mathbb{R}_{+}^{1} \stackrel{\text { def }}{\equiv}[0,+\infty)$ denotes the amount of accumulated in $i$-th $(i=\overline{1,(n-1)})$ seaport cargo which can be exported: $a_{i}$ does not contain the amount of cargo which was brought to $i$-th seaport from the seaports $\overline{1,(i-1)}$; $b_{i}^{k} \in \mathbb{R}_{+}^{1}$ denotes the total amount of cargo which can be exported from $i$-th $(i=\overline{1,(n-1)})$ seaport to $(i+1)$-th seaport by the $k$-th $(k=\overline{1, K})$ ship: as opposed to $a_{i}, b_{i}^{k}$ contains the cargo loaded to the ship $k$ in seaports $\overline{1,(i-1)} ; \quad x_{i, i+1}^{k} \in \mathbb{R}_{+}^{1}$ denotes the amount of loaded in $i$-th $(i=\overline{1,(n-1)})$ seaport cargo which has to be delivered by the $k$-th $(k=\overline{1, K})$ ship to $(i+1)$-th seaport. Obviously, $x \equiv\left(\begin{array}{ccccc}x_{1,2}^{1} & x_{2,3}^{1} & x_{3,4}^{1} & \ldots & x_{n-1, n}^{1} \\ x_{1,2}^{2} & x_{2,3}^{2} & x_{3,4}^{2} & \ldots & x_{n-1, n}^{2} \\ \ldots & \ldots & \ldots & \ldots & \ldots \\ x_{1,2}^{K} & x_{2,3}^{K} & x_{3,4}^{K} & \ldots & x_{n-1, n}^{K}\end{array}\right)$ is a full matrix with the size $K \times(n-1)$, called the plan of the transportation and containing all the variables $\left\{x_{i, i+1}^{k} \in \mathbb{R}_{+}^{1}\right\}_{i=1, \overline{1,(n-1)}}^{k=\overline{1, K}}$.

Thus, among the listed above parameters the desired are $\left\{x_{i, i+1}^{k} \in \mathbb{R}_{+}^{1}\right\}_{i=1, \overline{1,(n-1)}}^{k=\overline{1, K}}$, the amount of what, obviously, equals $K \cdot(n-1)$, but the other $\left[K \cdot\left(n^{2}-1\right)+n+3\right]$ parameters, included $T_{\text {start }}$ and $T_{\text {end }}$, are supposed to be given source data. The problem is in determination of a transportation plan $x \equiv\left\{x_{i, i+1}^{k} \in \mathbb{R}_{+}^{1}\right\}_{i=1, \overline{1, n-1)}}^{k=\overline{, K}}$, such that, firstly, the amount of transported cargo is maximal (first criterion), secondly, the total expenses of the transportation are minimal (second criterion) and, besides, generally speaking, these two criteria can be not equivalent.

\section{Construction of Mathematical Model}

Using introduced in the previous section denotations and assumptions, we will formulate the criteria for our problem. Obviously, the maximality criterion of the cargo transported by the $k$-th $(k=\overline{1, K})$ ship is the following function $\quad w_{1}^{k}(x) \stackrel{\text { def }}{\equiv} \sum_{i=1}^{n-1} x_{i, i+1}^{k}, \forall k=\overline{1, K}$. Therefore, the maximality criterion for all cargo transported by $K$ ships is determined by the additive function $\sum_{k=1}^{K} w_{1}^{k}$, so we have

$$
\max \left\{w_{1}(x) \stackrel{\text { def }}{\equiv} \sum_{i=1}^{n-1} \sum_{k=1}^{K} x_{i, i+1}^{k}\right\}
$$


For construction of the minimality criterion for the expenses of the transportation of the cargo transported by the $k$-th $(k=\overline{1, K})$ ship, it is necessary to calculate these expenses for every $i$-th $(i=\overline{1,(n-1)})$ seaport: for the Seaport \#1:

$$
\left(\sum_{j=2}^{n} c_{1, j}^{k}-10^{-2} \cdot \sum_{m=2}^{n-1} p_{1, m}^{k} \sum_{j=m+1}^{n} c_{1, j}^{k}\right) \cdot x_{1,2}^{k} ;
$$

etc;

for the Seaport \#(n- 2):

$$
\left(c_{n-2, n-1}^{k}+c_{n-2, n}^{k}-10^{-2} \cdot p_{n-2, n-1}^{k} \cdot c_{n-2, n}^{k}\right) \cdot x_{n-2, n-1}^{k}
$$

for the Seaport \#(n-1): $c_{n-1, n}^{k} \cdot x_{n-1, n}^{k}$.

Summing up these $(n-1)$ expressions, we get the minimality criterion for the expenses of the cargo transportation by the $k$-th $(k=\overline{1, K})$ ship:

$$
\begin{aligned}
& \min \left\{w _ { 2 } ^ { k } ( \tilde { x } ) \equiv \sum _ { i = 1 } ^ { \text { def } } \sum ^ { n - 1 } \left(\sum_{j=i+1}^{n} c_{i, j}^{k}\right.\right. \\
& \left.\left.-10^{-2} \cdot \sum_{m=i+1}^{n-1} p_{i, m}^{k} \sum_{j=m+1}^{n} c_{i, j}^{k}\right) \cdot x_{i, i+1}^{k}\right\}, \forall k=\overline{1, K}
\end{aligned}
$$

Remark 1. In Eq. (2) as well as in the future, if in an expression of the form $\sum_{i=i_{\text {satr }}}^{i_{\text {end }}} t(i)$ holds $i_{\text {start }}>i_{\text {end }}$, then this sum, as it is generally accepted in mathematics, will not be considered as an active one, so we will ignore it $[13,14]$. The end of the remark (EOR).

Therefore, the desired minimality criterion for the total expenses of cargo transportation performed by all $K$ ships is determined by the additive formula $\sum_{k=1}^{K} w_{2}^{k}$, i.e., the second criterion of the problem can be presented in the following form:

$$
\begin{aligned}
& \min \left\{w _ { 2 } ( x ) \equiv \sum _ { k = 1 } ^ { \operatorname { d e f } } \sum _ { i = 1 } ^ { K - 1 } \left(\sum_{j=i+1}^{n} c_{i, j}^{k}\right.\right. \\
& \left.\left.-10^{-2} \cdot \sum_{m=i+1}^{n-1} p_{i, m}^{k} \sum_{j=m+1}^{n} c_{i, j}^{k}\right) \cdot x_{i, i+1}^{k}\right\}
\end{aligned}
$$

Now we proceed to constructing of essential constraints of the problem. As the sum $\sum_{k=1}^{K} x_{i, i+1}^{k}$ is the amount of cargo which initially belonged to $i$-th $(i=\overline{1,(n-1)})$ seaport (implying that this has never been transported to this seaport form another) and which has to be transported to $(i+1)$-th seaport by the use of all $K$ ships, we can formulate the following $(n-1)$ inequalities:

$$
\sum_{k=1}^{K} x_{i, i+1}^{k} \leq a_{i}, \forall i=\overline{1,(n-1)}
$$

Then, as the amount of cargo transported between the seaports $i$ and $(i+1) \quad(i=\overline{1,(n-1)})$ by the $k$-th $(k=\overline{1, K})$ ship must not exceed the boat load $b_{i}^{k}$ of this ship, we can write:

for transport route "Seaport \#1 $\rightarrow$ Seaport \#2": $x_{1,2}^{k} \leq b_{1}^{k}$;

etc;

for transport route "Seaport \#( n-1) $\rightarrow$ Seaport \#n": $\sum_{j=1}^{n-1}\left(1-10^{-2} \cdot \sum_{m=1}^{n-j-1} p_{j, j+m}^{k}\right) \cdot x_{j, j+1}^{k}+x_{n-1, n}^{k} \leq b_{n-1}^{k}$.

Therefore, we have the following $K \cdot(i+1)$ inequalities:

$$
\begin{aligned}
& \sum_{j=1}^{i} x_{j, j+1}^{k}-10^{-2} \cdot \sum_{m=1}^{n-2} \sum_{j=1}^{i-m} p_{j, j+m}^{k} \cdot x_{j, j+1}^{k} \leq b_{i}^{k}, \\
& \forall(i=\overline{1,(n-1)} ; k=\overline{1, K})
\end{aligned}
$$

Finally, as $x_{i, i+1}^{k} \quad \forall(i=\overline{1,(n-1)} ; k=\overline{1, K})$ denotes the amount of cargo, we can write the following sign constraints:

$$
x_{i, i+1}^{k} \geq 0 \quad \forall(i=\overline{1,(n-1)}, k=\overline{1, K})
$$

Thus, combining the Eqs. (1) and (3)-(6), we get the following mathematical model of the considered problem: it is required to determine the values of the variables $\quad\left\{x_{i, i+1}^{k}\right\}_{i=1,(n-1)}^{k=\overline{1, K}} \quad$ which satisfies $(2 \cdot K+1) \cdot(n-1)$ constraints 


$$
\left\{\begin{array}{l}
\sum_{k=1}^{K} x_{i, i+1}^{k} \leq a_{i} \\
100 \cdot\left(\sum_{j=1}^{i} x_{j, j+1}^{k}-b_{i}^{k}\right)-\sum_{m=1}^{n-2} \sum_{j=1}^{i-m} p_{j, j+m}^{k} \cdot x_{j, j+1}^{k} \leq 0 \\
x_{i, i+1}^{k} \geq 0, \forall(i=\overline{1,(n-1)}, k=\overline{1, K})
\end{array}\right.
$$

maximize the criterion

$$
w_{1}(x) \stackrel{\text { def }}{\equiv} \sum_{i=1}^{n-1} \sum_{k=1}^{K} x_{i, i+1}^{k}
$$

and minimize the criterion

$$
\begin{aligned}
& w_{2}(x) \stackrel{\text { def }}{\equiv} \sum_{k=1}^{K} \sum_{i=1}^{n-1}\left(\sum_{j=i+1}^{n} c_{i, j}^{k}\right. \\
& \left.-10^{-2} \cdot \sum_{m=i+1}^{n-1} p_{i, m}^{k} \sum_{j=m+1}^{n} c_{i, j}^{k}\right) \cdot x_{i, i+1}^{k}
\end{aligned}
$$

Remark 2. It should be noted that after finding the values of the variables $\left\{x_{i, i+1}^{k}\right\}_{i=1, \overline{1,(n-1)}}^{k=\overline{1, K}}$ from the Models (7)-(9), from a practical point of view, instead of value $w_{2}$ from Model (9), the value $w_{2}^{k}$, determined by the Eq. (2) and presenting the expenses of transportation of cargo by the $k$-th $(k=\overline{1, K})$ ship, is of interest to us. EOR.

As the Models (7)-(9) is a multi-criterion problem, speaking about the solution of the Models (7)-(9), we will imply its Pareto optimal solution, which represents [15-20] generalization of concept of numerical function's optimum point for the case, when we have many function, notably, the solution of a multi-criterion problem is a Pareto optimal solution if the value of every criterion can be improved only at the cost of worsening the values of other criteria. The ultimate objective of this work consists in finding a transportation plan $\left\{x_{i, i+1}^{k}\right\}_{i=1, \overline{1,(n-1)}}^{k=\overline{1, K}}$ which is a Pareto optimal solution of the problem. We will shortly call this plan an effective plan. For achievement of the objective of this work, first of all, it is necessary to examine the optimality criterion for multi-criterion problems of linear programming. The following of this work is devoted to development of the required optimality conditions: firstly, for an arbitrary multi-criterion problem and, secondly, for bi-criterion problems in which the feasible solution set is a polyhedral set (i.e., consists of a finite system of inequalities) and one or both criteria can be non-linear.

Remark 3. In conclusion of this section, we emphasize that the formulated Criteria (8) and (9), generally speaking, can have different weights/importance/significance and, therefore, this circumstance should be taken into account during the process of solving the constructed model, if the decision maker misses information about the importance of some criteria. However, in this work, we will investigate the constructed Models (7)-(9), supposing that for the decision maker, Criteria (8) and (9) are equally important, i.e., the weighting coefficients of these criteria are equal. Therefore, without loss of generality, we can suppose that the weighting coefficients of both criteria are equal to one: if for some reasons it is required to interpret the weighting coefficients from a probabilistic point of view, then it will be easy to achieve them becoming less than one, notably, it will be sufficient to consider $\frac{\lambda_{1}}{\lambda_{1}+\lambda_{2}} \in(0,1)$ and $\frac{\lambda_{2}}{\lambda_{1}+\lambda_{2}} \in(0,1)$ instead of previous weighting coefficients $\lambda_{1} \in \mathbb{R}_{++}^{1}$ and $\lambda_{2} \in \mathbb{R}_{++}^{1}$ as the required weight criteria (in our case, supposing that $\lambda_{1}=\lambda_{2}$, it would be correct to assume that both weights are 0.5 . EOR.

\section{Optimality Conditions for Multi-criterion Linear Programming Problems}

Its well-known $[16,17]$ that in a multi-criterion problem of linear programming

$$
\begin{aligned}
& \max _{x \in X} / \min \left\{F(x) \stackrel{d e f}{\equiv}\left(\left\langle\alpha_{1}, x\right\rangle=y_{1}, \ldots,\left\langle\alpha_{m}, x\right\rangle=y_{m}\right)^{T}\right. \\
& \left\{X \stackrel{\text { def }}{\equiv}\left\{x \in \Omega \subseteq \mathbb{R}^{n}:\left\langle\beta_{j}, x\right\rangle \leq \gamma_{j} \in \mathbb{R}^{1}, j=\overline{1, L}\right\}\right. \\
& \Omega \stackrel{\text { def }}{\equiv}\left[x_{\min }, x_{\max }\right], x_{\min } \in \mathbb{R}^{n}, x_{\max } \in \mathbb{R}^{n} \\
& \alpha_{i} \in \mathbb{R}^{n}, i=\overline{1, m} ; \beta_{j} \in \mathbb{R}^{n}, j=\overline{1, L}
\end{aligned}
$$


where, $\langle\cdot, \cdot\rangle$ denotes inner product, the set of attainable vector estimates $Y(X) \stackrel{\text { def }}{\equiv}\left\{y \in \mathbb{R}^{m}: y=F(x), x \in X\right\}$ is not only a convex set, but also is a polyhedral set. Therefore, for every efficient estimate $y_{\text {efficient }}$, it is possible to find a vector $\lambda \in \Lambda_{++} \stackrel{\operatorname{def}}{\equiv}\left\{\lambda \in \mathbb{R}_{++}^{m}: \sum_{i=1}^{m} \lambda_{i}=1\right\}$, such that the point $y_{\text {efficient }}$ is the maximum/minimum point of function $\langle\lambda, y\rangle$ on the set $Y(X)$. So, because of a well-known theorem [21], mentioned below (see Theorem 1), we can say that the set $P(Y)$ of Pareto optimal estimate $[16,17]$ and the set $G(Y)$ of Geoffrion optimal estimate coincide (in other words, the properly efficient estimates) [22-26].

Theorem 1. Let $Y$ be an efficiently convex set. The estimate $\tilde{y}$ is a Geoffrion optimal estimate if and only if there exists a vector $\lambda \in \Lambda_{++}$, such that holds $\langle\lambda, \tilde{y}\rangle \geq\langle\lambda, y\rangle$ for $\forall y \in Y$. The end of the theorem (EOT).

Let's formulate and prove the following theorem.

Theorem 2. Implementation of equalities

$$
\begin{aligned}
& \langle\lambda, \alpha\rangle=\langle\theta, \beta\rangle \\
& \langle\theta, \gamma-\langle\beta, \tilde{x}\rangle\rangle=0
\end{aligned}
$$

for some vectors $\lambda \in \Lambda_{++}$and $\theta \in \mathbb{R}_{+}^{L}$ is necessary and sufficient for a point $\tilde{x}$ in a multi-criterion problem (10) of linear programming to be an efficient estimate (accordingly, to be a weakly efficient estimate (i.e., a Slater optimal estimate) if the stated equality holds for $\lambda \in \Lambda_{+} \stackrel{\text { def }}{\equiv}\left\{\lambda \in \mathbb{R}_{+}^{m}: \sum_{i=1}^{m} \lambda_{i}=1\right\}$ and $\theta \in \mathbb{R}_{+}^{L}$ ). EOT.

Proof of Theorem 2.

First of all, let's prove the necessity of implementation of Eqs. (11) and (12) if the point $\tilde{x}$ is efficient. To this effect, let's introduce the set $I[\tilde{x}] \stackrel{\text { def }}{\equiv}\left\{i \in\{1, \ldots, L\}:\left\langle\beta_{i}, \tilde{x}\right\rangle=\gamma_{i}\right\}$, which is called a set of indices of active essential constraints. It is not difficult to make sure that from the efficiency of the point $\tilde{x}$ follows inconsistence of the inequalities $\left\langle\alpha_{i}, x\right\rangle \geq 0, \forall i=\overline{1, m} ; \quad\left\langle\beta_{i}, x\right\rangle \leq 0, \forall i \in I[\tilde{x}]$. Indeed, supposing that the point $x \in \mathbb{R}^{n}$ satisfies these inequalities, we obtain that for $0<\varepsilon \ll 1$ and $\bar{x}=\tilde{x}+\varepsilon \cdot x$ the inequalities

$$
\begin{gathered}
\left\langle\alpha_{i}, \bar{x}\right\rangle \geq\left\langle\alpha_{i}, \tilde{x}\right\rangle, \forall i=\overline{1, m} \\
\left\langle\beta_{i}, \bar{x}\right\rangle \leq\left\langle\beta_{i}, \tilde{x}\right\rangle=\gamma_{i}, \forall i \in I[\tilde{x}] \\
\left\langle\beta_{i}, \bar{x}\right\rangle<\gamma_{i}, \quad \forall i \in\{1, \ldots, L\} / I[\tilde{x}]
\end{gathered}
$$

are true. Obviously, validity of these inequalities contradicts the efficiency of the point $\tilde{x}$, hence the inconsistence of the system $\left\langle\alpha_{i}, x\right\rangle \geq 0, \forall i=\overline{1, m}$; $\left\langle\beta_{i}, x\right\rangle \leq 0, \forall i \in I[\tilde{x}]$ is shown. Thus, by the well-known Tucker's theorem of the alternative [27-30], there exist vectors $\lambda \in \Lambda_{++}, \theta \in \mathbb{R}_{+}^{L}$, such that the equality $\sum_{i=1}^{m} \lambda_{i} \cdot \alpha_{i}=\sum_{i \in I \tilde{x}]} \theta_{i} \cdot \beta_{i}$ holds. This equality, obviously, is equivalent to Eqs. (11) and (12).

Now, we shall prove necessity of fulfillment of Eqs. (11) and (12) if the point $\tilde{x}$ is weakly efficient. Again, it is easy to make sure that from Slater optimality of the point $\tilde{x}$ follows inconsistence of the inequalities

$$
\left\langle\alpha_{i}, x\right\rangle>0, \forall i=\overline{1, m} ; \quad\left\langle\beta_{i}, x\right\rangle \leq 0, \forall i \in I[\tilde{x}]
$$

Thus, by Motzkin's theorem of the alternatives [31], there exist vectors $\lambda \in \Lambda_{+}, \theta \in \mathbb{R}_{+}^{L}$, such that the equality $\sum_{i=1}^{m} \lambda_{i} \cdot \alpha_{i}=\sum_{i \in I[\tilde{x}]} \theta_{i} \cdot \beta_{i}$, which is equivalent to Eqs. (11) and (12), holds. The proof of necessity of fulfillment of Eqs. (11) and (12) is complete. The proof of sufficiency of fulfillment of the conditions (11), (12) immediately follows from the Koopmans's lemma, which for the first time was obtained in Ref. [32] for linear problems of the special form. The proof of Theorem 2 is fully complete.

The results obtained above let us formulate the 
following important summary: in a multi-criterion problem of linear programming (10), the set $P(Y)$ $\left(P_{F}(X)\right)$ of efficient Pareto optimal solutions (estimate) and the set $G(Y)\left(G_{F}(X)\right)$ of Geoffrion optimal estimate (solutions) coincide, i.e., $P(Y)=G(Y)\left(P_{F}(X)=G_{F}(X)\right)$. In conclusion of this section, let's dwell on Pareto optimality conditions for bi-criterion problems of mathematical programming

$$
\left\{\begin{array}{l}
\sup _{x \in X}\left\{F(x) \stackrel{\text { def }}{\equiv}\left(f_{1}(x)=y_{1}, f_{2}(x)=y_{2}\right)\right\} \\
X \stackrel{\text { def }}{\equiv}\left\{x \in \Omega \subseteq \mathbb{R}^{n}: g_{j}(x) \leq \gamma_{j} \in \mathbb{R}^{1}, j=\overline{1, L}\right\} \\
\Omega \stackrel{\text { def }}{\equiv}\left[x_{\text {min }}, x_{\text {max }}\right], x_{\text {min }} \in \mathbb{R}^{n}, x_{\text {max }} \in \mathbb{R}^{n}
\end{array}\right.
$$

using prior information about the biggest and the smallest values of criteria in the set of efficient solutions.

Let $P_{F}(X) \neq \varnothing$, i.e., the set of Pareto optimal solution contains at least one point. Then, we can write that

$$
\sup _{x \in P_{F}(X)} f_{i}(x) \leq \sup _{x \in X} f_{i}(x), i=1,2,
$$

or $\sup _{x \in P_{F}(X)} f_{i}(x)=\sup _{x \in X} f_{i}(x), i=1,2$ in case of the set $P_{F}(X)$ being also an externally stable set [33]. Let's assume that $\tilde{y} \in P(Y), \quad \tilde{y}_{1}=\max _{y \in P(Y)} y_{1}$. In this case, we can state that $\tilde{y}_{2}=\min _{y \in P(Y)} y_{2}$. Indeed, if it is not true, then if $\tilde{y}_{2}>\inf _{y \in P(Y)} y_{2}$, there always exists a point $\hat{y} \in P(Y)$, such that $\tilde{y}_{2}>\hat{y}_{2}$. On the other hand, because of point's $\hat{y}$ efficiency, we can write $\tilde{y}_{2}<\hat{y}_{1}$, i.e., we obtain the inequality $\hat{y}_{1}>\tilde{y}_{2}>\hat{y}_{2}$ which contradicts the condition $\tilde{y} \in P(Y)$, $\tilde{y}_{1}=\max _{y \in P(Y)} y_{1}$, and, therefore, $\tilde{y}_{2}=\min _{y \in P(Y)} y_{2}$. We could note that this statement is not generalized for the problems of Eq. (13) in which the number of criteria is greater than three: for example, if in Eq. (13), there are three criteria and holds $Y=P(Y)$, then the set of Pareto efficient estimate will be a hexagon inscribed in the triangle with the vertices at the points $\tilde{y}_{1}, \tilde{y}_{2}, \tilde{y}_{3}$.

Now we suppose that in the problem (13) the set $X$ of feasible solutions is not, i.e., $X \neq \varnothing$, and consider the case, when $P_{F}(X) \neq \varnothing$. We introduce denotations $a_{2} \stackrel{\operatorname{def}}{\equiv} \sup _{x \in X^{*}} f_{2}(x)$ if $X^{*} \neq \varnothing, \quad$ or $\quad a_{2} \stackrel{\text { def }}{\equiv} \inf _{x \in X} f_{2}(x)$ if $X^{*}=\varnothing ; \quad b_{1} \stackrel{\text { def }}{\equiv} \sup _{x \in X} f_{1}(x), \quad b_{2} \stackrel{\text { def }}{\equiv} \sup _{x \in X} f_{2}(x)$, $X^{*} \stackrel{\text { def }}{\equiv}\left\{z \in X: f_{1}(z)=b_{1}\right\}$, and consider separately two possible cases: $X^{*}=\varnothing$ and $X^{*} \neq \varnothing$. If the first statement is true, then $f_{2}(x) \geq a_{2}$ for $\forall x \in P_{F}(X)$; if the second statement is true, then by virtue of the inequality (14), we have $f_{2}(x) \geq b_{2}$ and $f_{2}(x) \geq a_{2}$ for $\forall x \in P_{F}(X)$. Thus, we can state that for $\forall x \in P_{F}(X)$ holds $f_{2}(x) \in\left[a_{2}, b_{2}\right]$ (here the segment $\left[a_{2}, b_{2}\right]$ should be considered conditionally, notably, if one or both boundaries of the segment are not limited, then it should be considered as a ray or real axes). Now we can formulate and investigate the following important scalar problem:

$$
\left\{\begin{array}{l}
\max _{x \in X}\left\{f_{1}(x)\right\} \\
f_{2}(x) \geq \omega \in\left[a_{2}, b_{2}\right]
\end{array}\right.
$$

Let's prove that if the problem (15) has unique solution (with an accuracy up to the equivalence) $\tilde{x}$, then this solution is an efficient solution of the problem (13), and vice versa. Indeed, let $\tilde{x}$ be an efficient solution of the problem (13), i.e., $\tilde{x} \in P_{F}(X)$. Then, by the Podinovsky's theorem [34], we obtain that $\tilde{x}$ is a solution of the problem (15) with $\omega=f_{2}(\tilde{x}) \in\left[a_{2}, b_{2}\right]$. Now let $\tilde{x}$ be the unique solution (with an accuracy up to the equivalence) of the problem (15). Then, from two theorems given below immediately follows [35] efficiency of the point $\tilde{x}$ in the problem (13) if in these theorems we substitute $p$ for $1, \varphi_{0}(f(y))$ for $f_{1}(x), \varphi_{1}(f(y))$ for $f_{2}(x)$, $t_{1}$ for $\omega, y$ for $x, y^{0}$ for $\tilde{x}$. 
Theorem 3. Let $\varphi_{0}$ be an increasing on the set $Y \stackrel{\text { def }}{\equiv}\left\{y \in \mathbb{R}^{m}: y=F(x), x \in X\right\}$ of attainable vector estimates function; functions $\varphi_{j}(j=\overline{1, p})$ are non-decreasing on the set $Y$ functions. If the estimate $y^{0} \in Z$, where

$$
Z \stackrel{\text { def }}{\equiv}\left\{y \in Y: \varphi_{j}(y) \geq t_{j}, j=\overline{1, p}\right\}
$$

and $t_{j}$ are arbitrary fixed numbers, satisfies the condition

$$
y^{0}=\arg \max _{y \in Z} \varphi_{0}(y)
$$

then it is weakly efficient. EOT.

Theorem 4. Let $\varphi_{j}(j=\overline{0, p \geq 1})$ be non-decreasing on the set $Y$ functions. If the point $y^{0} \in Z$, where the set $Z$ is defined according to Eq. (16), satisfies the condition (17), then for its efficiency is sufficient fulfillment of one of the following conditions: $\varphi_{0}$ is increasing on the set $Z ; y^{0}$ is the only maximum point of the function $\varphi_{0}$ in the set $Z$. EOT.

Thus, concerning the problem (15), we can state that $\tilde{x} \in P_{F}(X)$, if and only if the point $\tilde{x}$ is the unique solution (with accuracy up to the equivalence) of the problem (15) with $\omega \in\left[a_{2}, b_{2}\right]$. Besides, now it is obvious that the least segment of the parameter $\omega$ variation with which the statement about one-to-one correspondence between the efficient points of the vector problem (13) and the solution of the scalar problem (15) stays true, is the segment $\left[\inf _{x \in P_{F}(X)} f_{2}(x), \sup _{x \in P_{F}(X)} f_{2}(x)\right]$. Constructing of the least segment is important, because its absence would permit the criteria to stay underattainable or overattainable. However, solving many particular problems does not work well to determine (or at least, it appears to be a laborious process) the desired least segment, so instead of it, as a rule, a segment with "safety boundaries" is taken, which adds undesirable arbitrariness to the scientifically substantiated procedure of efficient decision making.
In the next section of this work, one well-known method of contraction of the Pareto set (it will be recalled that the Pareto set (so-called the Pareto boundary) is the set of rate points at which it is impossible to improve any of the rates without simultaneously decreasing at least one of the others) will be introduced: namely, the method called "goal attainment method" developed by F. W. Gembicki [36-39] will be used for solving of the model constructed in Eqs. (7)-(9).

\section{Some Basic Approaches of Contraction of the Pareto Boundary}

Without loss of generality, in this section, we will investigate only a multi-criterion problem:

$$
\left\{\begin{array}{l}
\min _{x \in X}\left\{F(x) \stackrel{\text { def }}{\equiv}\left(f_{1}(x)=z_{1}, \ldots, f_{m}(x)=z_{m}\right)^{T}\right\} \\
X \stackrel{\text { def }}{\equiv}\left\{x \in \Omega: g_{i}(x) \leq 0, i \in I_{1} ; g_{i}(x)=0, i \in I_{2}\right\} \\
\Omega \stackrel{\text { def }}{\equiv}\left[x_{\min }, x_{\max }\right] \subseteq \mathbb{R}^{n}, x_{\min } \in \mathbb{R}^{n}, x_{\max } \in \mathbb{R}^{n} \\
I_{1} \cup I_{2}=I, I_{1} \cap I_{2}=\varnothing,|I|=L
\end{array}\right.
$$

and expounded method of contraction of the Pareto set will consider only for it.

The point of the goal attainment method for the first time offered by F. W. Gembicki in his Ph.D. thesis [36] consists of the following: a set $\left\{f_{1}^{*}, \ldots, f_{m}^{*}\right\}$ called a set of intentions and connected to the objective vector $F(x)=\left(f_{1}(x), \ldots, f_{m}(x)\right)^{T} \quad$ for the initial multi-criterion problem (18) is formulated. We should note that as $f_{i}^{*}(i=\overline{1, m})$ can be taken the optimal value of the $i$-th $(i=\overline{1, m})$ composite one-criterion problem:

$$
\left\{\begin{array}{l}
\min _{x \in X}\left\{f_{i}(x)\right\} \\
X \stackrel{\text { def }}{\equiv}\left\{x \in \Omega: g_{i}(x) \leq 0, i \in I_{1} ; g_{i}(x)=0, i \in I_{2}\right\} \\
\Omega \stackrel{\text { def }}{\equiv}\left[x_{\min }, x_{\max }\right] \subseteq \mathbb{R}^{n}, x_{\min } \in \mathbb{R}^{n}, x_{\max } \in \mathbb{R}^{n} \\
I_{1} \cup I_{2}=I, I_{1} \cap I_{2}=\varnothing,|I|=L
\end{array}\right.
$$

Formulating of the set of intentions $\left\{f_{1}^{*}, \ldots, f_{m}^{*}\right\}$ 
means assumption to the effect that some or even all objectives can be underattainable as well as overattainable, which lets researcher express his intentions relatively precisely. Moreover, the relative degree of underattainability or overattainability of the formulated intentions is controlled using $R \cdot \omega$, where the scalar parameter $R \in \mathbb{R}^{1}$ denotes unknown/desired relaxation of the intention; vector parameter $\omega \in \mathbb{R}^{m}$ denotes vector of weighted coefficients. In the case of underattainment of the desired goals, the smaller weighting coefficient is associated with the more important objectives. For over-attainment of the desired goals, the smaller weighting coefficient is associated with the less important objectives. Now, the point of the magnitude $R \cdot \omega$ is absolutely obvious: it shows that rigidity of the intention $F^{*}=\left\{f_{1}^{*}, \ldots, f_{m}^{*}\right\}$.

Mathematical formulation of the goal attainment method, which allows us to go to the problem of vector optimization (18), has the following form:

$$
\left\{\begin{array}{l}
\min _{x \in S}\{R\} \\
S \stackrel{\text { def }}{\equiv}\left\{x \in \Omega: f_{i}(x)-R \cdot \omega_{i} \leq f_{i}^{*}, i=\overline{1, m}\right. \\
\left.g_{i}(x) \leq 0, i \in I_{1} ; g_{i}(x)=0, i \in I_{2}\right\} \\
\quad \stackrel{\text { def }}{\equiv}\left[x_{\min }, x_{\max }\right] \subseteq \mathbb{R}^{n}, x_{\min } \in \mathbb{R}^{n}, x_{\max } \in \mathbb{R}^{n} \\
I_{1} \cup I_{2}=I, I_{1} \cap I_{2}=\varnothing,|I|=L
\end{array}\right.
$$

Controlling the weighted coefficients $\omega_{i} \in \mathbb{R}^{1}(i=\overline{1, m})$, researcher gets the opportunity to influence the extent of interconnection between the criteria of the problem (18). For instance, substituting the elements of the vector $\omega \in \mathbb{R}^{m}$ for the values of the corresponding coordinates of the initial vector of intentions $F^{*}=\left\{f_{1}^{*}, \ldots, f_{m}^{*}\right\}$ will mean that the researcher desires to achieve the same extent (for example, in percent) of under-attainability or over-attainability of all objectives. Setting some elements of the vector $\omega \in \mathbb{R}^{m}$ zero value, it is possible to add to the problem some rigid constraints.
The goal attainment method ensures a good intuitive interpretation of the considered problem, which is completely decidable by standard optimization procedures. That is why this method has well-earned popularity. In the works [38, 40, 41], an example of successful use of goal attainment method for solving multi-criterion problems of the control theory is clearly demonstrated. However, this method has the same disadvantages as those of the goal programming proposed in the work [42] (this very important method has been further developed in Refs. [43-47] and other works). Namely, the preferred solution is sensitive to the goal vector and the weighting vector given by the decision maker. When some of the desired goals are underattained and some overattained, the goal attainment method has difficulty in determining the proper weighting coefficient $\omega_{i}(i=\overline{1, m})$. However, as opposed to goal programming method, many models obtained by the goal attainment method, have fewer variables to work with, so it will be computationally faster. The goal attainment method for two objectives is illustrated in Fig. 5.

\section{Solving the Proposed Models (7)-(9) and (2), (7), (8) on the Assumption of Equivalence of Criteria}

In this section, we will investigate bi-criterion (7)-(9) and $(K+1)$-criterion problems (2), (7), (8) introduced in the third section. First of all, we will try to introduce and explain a transformation which sets bi-univocal correspondence between the three-index desired variables $\quad x_{i, i+1}^{k}(k=\overline{1, K} ; i=\overline{1,(n-1)})$ and new one-index variables $y_{j}(j=\overline{1, K \cdot(n-1)})$, using which, because of many reasons (not only with the objective of simplification of the variables appearance), is rational to reformulate and investigate Models (7)-(9) and (2), (7), (8).

So, instead of the variables $x_{i, i+1}^{k}(k=\overline{1, K} ; i=\overline{1,(n-1)})$ we will introduce new 


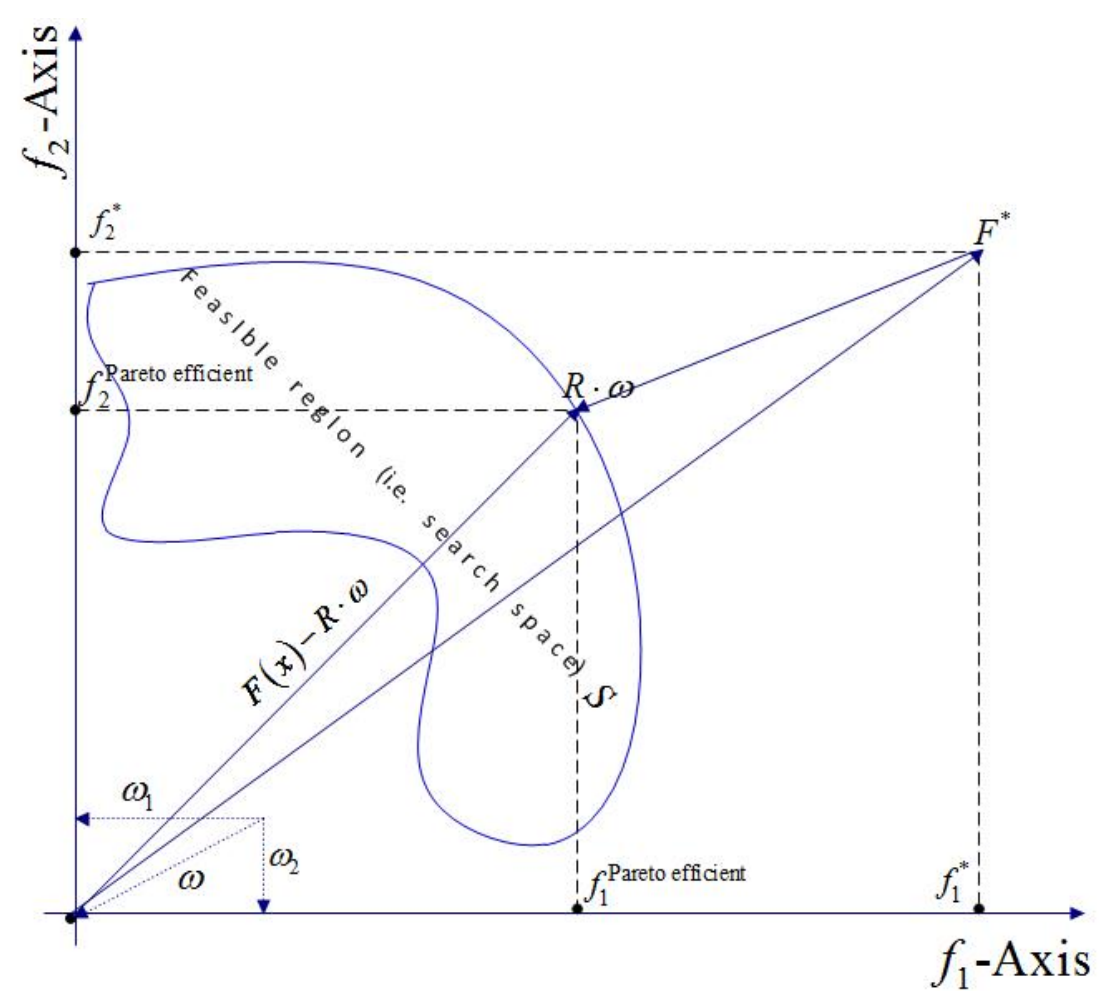

Fig. 5 Illustration of the goal attainment method for two objectives: the direction of the preferred solution vector $(F(x)-R \cdot \omega)$ is fixed by the goal vector $F^{*}$ and the weight vector $\omega$. The minimum value of $R$ occurs where $(F(x)-R \cdot \omega)$ vector intersects the upper bound of the feasible solution set $S$ (for maximizing $F(x)$ ).

variables $y_{j}(j=1, K \cdot(n-1))$, where index $j \in\{1,2, \ldots, K \cdot(n-1)\}$ of the variable $y_{j}$ and indexes $i, k(i \in\{1,2, \ldots, n-1\} ; k \in\{1,2, \ldots, K\})$ of the variable $x_{i, i+1}^{k}$ are connected by the following relations:

$j=(n-1) \cdot(k-1)+i$ for each fixed pair $i, k$

$$
\left\{\begin{array}{l}
i \equiv j(\bmod (n-1)) \\
k=1+\frac{j-i}{n-1}
\end{array} \text { for each fixed } j(22)\right.
$$

The role of Transformation (21) consists in the following: for each fixed pair of indexes, $i, k$ of the variable $x_{i, i+1}^{k} \quad$ Transformation (21) puts in correspondence unique index $j$ of the variable $y_{j}$. In other words, Transformation (21) uniquely maps (uniqueness is obvious as index $j$ runs over the rows of the matrix $x$ ) the three-index variable $x_{i, i+1}^{k}$ into one-index variable $y_{j}: \quad x_{i, i+1}^{k} \stackrel{(21)}{\rightarrow} y_{j}=y_{i+(k-1) \cdot(n-1)}$. Therefore, instead of the desired plan of transportation, which has the form of the following matrix:

$$
x \equiv\left(\begin{array}{ccccc}
x_{1,2}^{1} & x_{2,3}^{1} & x_{3,4}^{1} & \ldots & x_{n-1, n}^{1} \\
x_{1,2}^{2} & x_{2,3}^{2} & x_{3,4}^{2} & \ldots & x_{n-1, n}^{2} \\
\ldots & \ldots & \ldots & \ldots & \ldots \\
x_{1,2}^{K} & x_{2,3}^{K} & x_{3,4}^{K} & \ldots & x_{n-1, n}^{K}
\end{array}\right)
$$

we obtain the same plan of transportation in the form of a column-vector $y \equiv\left(y_{1}, y_{2}, \ldots, y_{K \cdot(n-1)}\right)^{T}$.

Transformation (22) is inverse transformation to Transformation (21). Notably, for each fixed index $j$ of the variable $y_{j}$, Transformation (22) puts in correspondence an unique pair of indexes $i, k$ of the variable $x_{i, i+1}^{k}$. Let's prove that Transformation (22), firstly, returns us positive integers $i, k$; and, secondly, unambiguously determines the pair of indexes $i, k$.

First of all, let's prove the naturalness of the numbers $i, k$. Naturalness of $i$ immediately follows from the first formula of Transformation (22), which is nothing else than the definition of congruence $(n-1)$ between 
numbers $i$ and $j[48,49]$. Now let's prove that $k \in \mathbb{N}$. As $j \in \mathbb{N}$, it is obvious, that there always exist numbers $m_{1} \in \mathbb{N} \cup\{0\}, \quad m_{2} \in \mathbb{N}, \quad$ such that $j=(n-1) \cdot m_{1}+m_{2}$. Then, from $i \in\{1,2, \ldots, n-1\}$ and $i \equiv j(\bmod (n-1)) \quad$ follows that $i=m_{2}$, i.e. $j=(n-1) \cdot m_{1}+i$. As from the uniquely forward Transformation (21) follows unique determination of the index $k$, we can write that $k=1+\frac{j-i}{n-1}=1+\frac{(n-1) \cdot m_{1}+i-i}{n-1}=1+m_{1}$. As $m_{1} \in \mathbb{N}$, from the last expression follows $k=\left(1+m_{1}\right) \in \mathbb{N}$. The proof of naturalness of the indices pair $i, k$ determined by the transformation (22) is complete.

Now we have to prove that the choice of natural indexes $i, k$ by Eq. (22) is unique. The proof of this statement will be based on the definition of the complete residue class modulo $m$ concept [48, 49]: the set $A$ is called a complete residue class modulo $m$, if for $\forall l \in\{0,1,2, \ldots, m-1\}$, there exists an element $a \in A$, such that $l \equiv a(\bmod m)$. As for out matrix $x$ (i.e., the transportation plan), the column index $i$ varies from 1 to $(n-1)$ included, the set $\{1,2, \ldots, n-1\}$ is a complete residue class modulo $(n-1)$, i.e., for every two different elements of the set $\{1,2, \ldots, n-1\}$, the remainders of their division by $(n-1)$ are different too:

$$
\forall i_{1}, \forall i_{2} \in\{1,2, \ldots, n-1\}: i_{1} \neq i_{2} \Rightarrow i_{1} \not i_{2}(\bmod (n-1))
$$

In other words, we have proved the uniqueness of the index $i$. In view of the fact that with fixed values of $j$ and $n$, the desired index $k$, determined by the second formula of Transformation (22) is a single-valued function of an argument $i$ from the proven above fact about the uniqueness of the index $i$ follows the uniqueness of the index $k$. The proof of the inverse Transformation (22) is complete.

Thus, in future we will use new variables $\left\{y_{j}\right\}_{j=1, K \cdot(n-1)}$ instead of the old variables $\left\{x_{i, i+1}^{k}\right\}_{i=1,(n-1)}^{k=\overline{1, K}}$.
6.1 Transforming the Bi-criterion Problem (7)-(9) into a Simple Linear Programming Problem

Let's reformulate modelS (7)-(9), using new variables $\left\{y_{j}\right\}_{j=1, K \cdot(n-1)}$, in which the index $j$ is determined in accordance with Transformation (21):

$$
\begin{gathered}
\max \left\{w_{1}(y) \stackrel{\operatorname{def}}{\equiv} \sum_{j=1}^{K \cdot(n-1)} y_{j}\right\} \\
\min \left\{w_{2}(y) \equiv \sum_{j=1}^{\operatorname{def}} \xi_{j}^{K \cdot(n-1)} \cdot y_{j}\right\}
\end{gathered}
$$

subject to:

$$
\left\{\begin{array}{l}
\sum_{j=1}^{K} y_{i+(j-1) \cdot(n-1)} \leq a_{i} \\
\sum_{m=1}^{i-1} \pi_{i}^{k}(m) \cdot y_{m+(k-1) \cdot(n-1)}+y_{i+(k-1) \cdot(n-1)} \leq b_{i}^{k} \\
y_{j} \geq 0 \\
\forall(i=\overline{1,(n-1)} ; j=\overline{1, K \cdot(n-1)} ; k=\overline{1, K})
\end{array}\right.
$$

where, for each value of $i \quad(i=\overline{2,(n-1)})$ and $k$ $(k=\overline{1, K})$, the coefficients $\pi_{i}^{k}(m)$, which appear in the second line of the constraints system (25) are calculated using the following formula:

$$
\pi_{i}^{k}(m) \stackrel{\text { def }}{\equiv} 1-10^{-2} \cdot \sum_{j=m+1}^{i} p_{m, j}^{k}
$$

the coefficients $\left\{\xi_{j}\right\}_{j=1, K \cdot(n-1)}$ in Criterion (24) are elements of a row-vector $\xi$ with the size $1 \times K \cdot(n-1)$, which is created applying Transformation (21) to the matrix $\eta \in \mathbb{R}_{+}^{1}(K \times(n-1))$ whose elements $\left\{\eta_{k, i}\right\}_{k=1, K}^{i=1,(n-1)}$ are calculated using the following formula:

$$
\begin{aligned}
& \eta_{k, i} \stackrel{\text { def }}{\equiv}-10^{-2} \cdot \sum_{m=i+1}^{n-1} p_{i, m}^{k} \sum_{j=m+1}^{n} c_{i, j}^{k} \\
& +\sum_{j=i+1}^{n} c_{i, j}^{k}, \forall(k=\overline{1, K}, i=\overline{1,(n-1)})
\end{aligned}
$$

Thus, instead of initial bi-criterion Models (7)-(9), we have obtained an equivalent bi-criterion problem (23)-(27), for which finding of the Pareto optimal 
solution will be achieved by using Gembicki goal attainment method, which was described in Section 5.3 of this work.

We introduce the denotation

$$
\begin{aligned}
& Y_{(25)} \stackrel{\text { def }}{\equiv}\left\{y \in \mathbb{R}_{+}^{1}: \sum_{j=1}^{K} y_{i+(j-1) \cdot(n-1)} \leq a_{i}\right. \\
& \sum_{m=1}^{i-1} \pi_{i}^{k}(m) \cdot y_{m+(k-1) \cdot(n-1)}+y_{i+(k-1) \cdot(n-1)} \leq b_{i}^{k} \\
& \forall(i=\overline{1,(n-1)} ; k=\overline{1, K})
\end{aligned}
$$

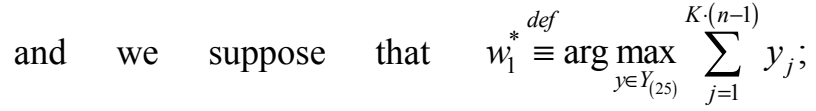
$w_{2}^{*} \stackrel{\operatorname{def}}{\equiv} \arg \min _{y \in Y_{(25)}} \sum_{j=1}^{K \cdot(n-1)} \xi_{j} \cdot y_{j}, \quad$ i.e., $\quad w_{1}^{*} \quad$ and $w_{2}^{*}$ are optimal solutions of the composite one-criterion problems (23), (25), (26) and (24)-(27), respectively. Then, by virtue of the results of the previous sections, we can state that Pareto optimal solution of the problem (23)-(27) (therefore, of the initial problem (7)-(9), due to one-to-one transformations $(21),(22))$ is the optimal solution of the following one-criterion problem of linear programming:

$$
\begin{aligned}
& \min \left\{M_{1} \cdot \sum_{i=1}^{(K+1) \cdot(n-1)+2} z_{i+(2 \cdot K+1) \cdot(n-1)+2}\right. \\
& \left.+z_{i+(3 \cdot K+2) \cdot(n-1)+5}-z_{i+(3 \cdot K+2) \cdot(n-1)+6}\right\}
\end{aligned}
$$

subject to:

$$
\left\{\begin{array}{l}
\sum_{m=1}^{i-1} \pi_{i}^{k}(m) \cdot z_{m+(k-1) \cdot(n-1)}+z_{i+(k-1) \cdot(n-1)}+z_{i+(K+k) \cdot(n-1)} \\
+z_{i+(2 \cdot K+k+1) \cdot(n-1)+2}=b_{i}^{k}, \forall(i=\overline{1,(n-1)} ; k=\overline{1, K}) \\
K \cdot(n-1) \\
\sum_{j=1}^{*} z_{j}-z_{(2 \cdot K+1) \cdot(n-1)+1}+z_{(3 \cdot K+2) \cdot(n-1)+3} \\
+w_{1}^{*} \cdot z_{i+(3 \cdot K+2) \cdot(n-1)+5}-w_{1}^{*} \cdot z_{i+(3 \cdot K+2) \cdot(n-1)+6}=w_{1}^{*} \\
K \cdot(n-1) \\
\sum_{j=1}^{*} \xi_{j} \cdot z_{j}+z_{(2 \cdot K+1) \cdot(n-1)+2}+z_{(3 \cdot K+2) \cdot(n-1)+4} \\
-w_{2}^{*} \cdot z_{i+(3 \cdot K+2) \cdot(n-1)+5}+w_{2}^{*} \cdot z_{i+(3 \cdot K+2) \cdot(n-1)+6}=w_{2}^{*} \\
z_{j} \geq 0, \forall j=1, \overline{1((3 \cdot K+2) \cdot(n-1)+6)}
\end{array}\right.
$$

where, $\quad z \stackrel{\text { def }}{\equiv}\left\{z_{i} \in \mathbb{R}_{+}^{1}\right\}_{i=1,((3 \cdot K+2) \cdot(n-1)+6)}$ is an introduced for the convenience column-vector, first $K \cdot(n-1)$ elements of what form the desired vector $y$ of the problem (23)-(27), i.e., $z_{i}=y_{i}$ for $\forall i=\overline{1, K \cdot(n-1)}$; as $M$ in Eq. (29), we can choose sufficiently great number, for example, the following number:

$$
M_{1} \stackrel{\text { def }}{\equiv} K+\max \left\{1 ;\left|w_{1}^{*}\right| ;\left|w_{2}^{*}\right| ; \underset{i=1,(n-1)}{\max }\left\{\left|a_{i}\right|\right\}\right.
$$

$$
\left.\max _{\substack{i=1,(n-1) \\ k=1, K}}\left\{\left|b_{i}^{k}\right|\right\} ; \max _{\substack{k=1, K \\ i=2,(n-1) \\ m=1,(i-1)}}\left\{\left|\pi_{i}^{k}(m)\right|\right\} ; \max _{\substack{k=1, K \\ i=1,(n-1)}}\left\{\left|\eta_{k, i}\right|\right\}\right\}
$$

Remark 5. It is not difficult to see that the one-criterion problem (29)-(31) is formulated in the form of the so-called Big M Method [50]. Obviously, with $\quad w_{i}^{*} \geq 0(i=1,2)$ the point $z=(\underbrace{0, \ldots, 0,}_{(2 \cdot K+1) \cdot(n-1)+2} \underbrace{a_{1}, \ldots, a_{n-1}}_{n-1}$ $\underbrace{b_{1}^{1}, b_{2}^{1}, \ldots, b_{n-1}^{K}}_{K \cdot(n-1)}, w_{1}^{*}, w_{2}^{*}, 0,0)^{T} \in \mathbb{R}_{+}^{(3 \cdot K+2) \cdot(n-1)+6}$ is an extreme point of the convex set, determined by the system (30). Exactly, this point should be chosen as the origin extreme point for applying the simplex algorithm. As a strict explanation of the Big M Method as well as the conditions of its use can be found, for instance, in the works $[50,51]$, we will not concern these questions in our work. EOR.

Remark 6. In the problems (29)-(31), the number of the desired variables is $((3 \cdot K+2) \cdot(n-1)+6)$; the number of essential constraints is $((K+1) \cdot(n-1)+2)$; the number of the source data is $\left(\frac{(n-1) \cdot(3 \cdot n+4) \cdot K}{2}+n+5\right),\left(\left(n^{2}-1\right) \cdot K+n+2\right)$ of which have to be given a priori and 
$\left(\frac{(n-1) \cdot(n+2) \cdot K}{2}+3\right)$ have to be calculated

beforehand. Therefore, the investigated problem can be considered as a large-scale problem: for instance, even with scanty data (from the point of view of modeling and solving of real-life practical problems in the modern transport logistics) $K=30, n=100$, we will have to solve an one-criterion problem (29)-(31) with 9,114 variables, 3,071 essential constraints and 451,545 source data. That is why the idea of using not traditional simplex method, but one of its modified variants or decomposition algorithms [52-56], applying the parallelizing technique [57-62] as well as high-performance computing, for solving the problem (29)-(31) certainly makes sense. These special algorithms, as a rule, let make the problem solving substantially less computationally intensive and to compactly place the information in the computer's memory. EOR.

So, let the vector $z^{\text {opt. }} \in \mathbb{R}_{+}^{(3 \cdot K+2) \cdot(n-1)+6}$ be the solution of the problems (29)-(31), found but a decomposition algorithm or the parallelizing realization of the simplex method. Then, $y^{\text {Pareto }}=\left(z_{1}^{\text {opt. }}, \ldots, z_{K \cdot(n-1)}^{\text {opt. }}\right)$ is a trade-off solution of the bi-criterion problems (23)-(27). Therefore, by using the inverse transformation (22), we can uniquely determine Pareto optimal transportation plan $x^{\text {Pareto }} \in \mathbb{R}_{+}^{1}(K \times(n-1))$ :

$$
x^{\text {Pareto }}=\left(\begin{array}{ccc}
z_{1}^{\text {opt. }} & \ldots & z_{n-1}^{\text {opt. }} \\
\ldots & \ldots & \ldots \\
z_{(K-1) \cdot(n-1)+1}^{\text {opt. }} & \ldots & z_{K \cdot(n-1)}^{\text {opt. }}
\end{array}\right)
$$

6.2 Transforming the $(K+1)$-Criterion Problems (2), (7), (8) into a Simple Linear Programming Problem

By analogy with Section 6.1, we, firstly, reformulate the $(K+1)$-criterion problems (2), (7), (8) in new variables $\left\{y_{j}\right\}_{j=1, K \cdot(n-1)}$, in which the index $j$ is calculated in accordance with Transformation (21), and then reduce the obtained $(K+1)$-criterion problem to a simple linear programming problem using the Big $M$ Method once again.

Thus, in new variables, we have the following $(K+1)$-criterion problem:

$$
\begin{gathered}
\max \left\{w_{1}(y) \stackrel{\operatorname{def}}{\equiv} \sum_{j=1}^{K \cdot(n-1)} y_{j}\right\} \\
\min \left\{w_{2}^{k}(\tilde{x}) \stackrel{\text { def }}{\equiv} \sum_{i=1}^{n-1} \eta_{k, i} \cdot y_{i+(k-1) \cdot(n-1)}\right\}, \forall k=\overline{1, K}
\end{gathered}
$$

subject to:

$$
\left\{\begin{array}{l}
\sum_{j=1}^{K} y_{i+(j-1) \cdot(n-1)} \leq a_{i} \\
\sum_{m=1}^{i-1} \pi_{i}^{k}(m) \cdot y_{m+(k-1) \cdot(n-1)}+y_{i+(k-1) \cdot(n-1)} \leq b_{i}^{k} \\
y_{j} \geq 0 \\
\forall(i=\overline{1,(n-1)} ; j=\overline{1, K \cdot(n-1)} ; k=\overline{1, K})
\end{array}\right.
$$

where, $\pi_{i}^{k}(m)$ and $\eta_{k, i}$ are calculated using, respectively, Eqs. (26) and (27).

As it was done in the previous subsection, we use the goal attainment method of Gembicki as well as the Big M Method, but now instead of the $(K+1)$-criterion problems (33)-(35), we will have the following one-criterion problem:

$$
\begin{aligned}
& \min \left\{M_{2} \cdot \sum_{i=1}^{(K+1) \cdot n} z_{i+K \cdot(2 \cdot n-1)}\right. \\
& \left.+z_{(3 \cdot K+1) \cdot n-K+1}-z_{(3 \cdot K+1) \cdot n-K+2}\right\}
\end{aligned}
$$

subject to:

$$
\begin{aligned}
& \sum_{j=1}^{K} z_{i+(j-1) \cdot(n-1)}+z_{i+K \cdot(n-1)} \\
& +z_{i+K \cdot(2 \cdot n-1)}=a_{i}, \quad \forall i=\overline{1,(n-1)}, \\
& \sum_{m=1}^{i-1} \pi_{i}^{k}(m) \cdot z_{m+(k-1) \cdot(n-1)} \\
& +z_{i+(k-1) \cdot(n-1)}+z_{i+(K+k) \cdot(n-1)} \\
& +z_{i+(K+k) \cdot(n-1)+n \cdot K}=b_{i}^{k}, \\
& \forall(i=\overline{1,(n-1)} ; \quad k=\overline{1, K}),
\end{aligned}
$$




$$
\begin{aligned}
& \sum_{j=1}^{K \cdot(n-1)} z_{j}-z_{(2 \cdot K+1) \cdot(n-1)+1} \\
& +z_{(3 \cdot K+1) \cdot n-2 \cdot K}+w_{1}^{*} \cdot z_{(3 \cdot K+1) \cdot n-K+1} \\
& -w_{1}^{*} \cdot z_{(3 \cdot K+1) \cdot n-K+2}=w_{1}^{*}, \\
& \sum_{i=1}^{n-1} \eta_{k, i} \cdot z_{i+(k-1) \cdot(n-1)}+z_{2 \cdot K \cdot(n-1)+n} \\
& +z_{k+(3 \cdot K+2) \cdot n-2 \cdot K}-w_{2}^{k^{*}} \cdot z_{(3 \cdot K+1) \cdot n-K+1} \\
& +w_{2}^{k^{*}} \cdot z_{(3 \cdot K+1) \cdot n-K+2}=w_{2}^{k^{*}}, \forall k=\overline{1, K,} \\
& \quad z_{j} \geq 0, \forall j=\overline{1,((3 \cdot K+1) \cdot n-K+2)}
\end{aligned}
$$

where,

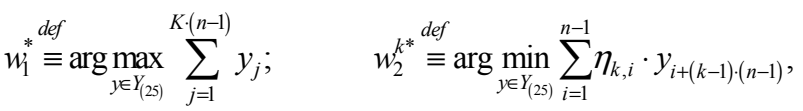
$\forall k=\overline{1, K}$, the set $Y_{(25)}$ is determined by Eq. (28); the number $M$ is sufficiently great and can be considered to be, for example,

$$
\begin{gathered}
M_{2} \stackrel{\text { def }}{\equiv} K+\max \left\{1 ;\left|w_{1}^{*}\right| ; \max _{k=1, K}\left|w_{2}^{k^{*}}\right| ; \max _{i=1,(n-1)}\left\{\left|a_{i}\right|\right\}\right. \\
\left.\max _{\substack{i=1,(n-1) \\
k=1, K}}\left\{\left|b_{i}^{k}\right|\right\} ; \max _{\substack{k=1, K \\
i=2,(n-1) \\
m=1,(i-1)}}\left\{\left|\pi_{i}^{k}(m)\right|\right\} ; \max _{\substack{k=1, K \\
i=1,(n-1)}}\left\{\left|\eta_{k, i}\right|\right\}\right\}
\end{gathered}
$$

Mentioned in Remarks 5 and 6, inferences with the corresponding obvious corrections of quantitative type stay right for Problems (36)-(38), too.

Supposing that the vector $z^{\text {opt. }} \in \mathbb{R}_{+}^{(3 \cdot K+1) \cdot n-K+2}$ is the solution of Problems (36)-(38), the desired compromise plan of transportation for the initial model (2), (7), (8) is the matrix $x^{\text {Pareto }} \in \mathbb{R}_{+}^{1}(K \times(n-1))$ of Eq. (32).

\section{Ill-Posedness Research and Construction of the Regularized Solution}

As it is well known [63-65], a mathematical problem which corresponds to physical or economic, etc. reality, has to satisfy the following basic requirements:

- The solution must exist;

- The solution should be uniquely determined;
- The solution should depend continuously on the data (requirement of stability).

The first requirement expresses the logical condition that not too much, i.e., no mutually contradictory properties, is demanded of the solution. The second requirement stipulates completeness of the problem: leeway or ambiguity should be excluded unless inherent in the physical or economic, etc. situation (cases in which uniqueness is not a proper requirement do occur). The third requirement, particularly incisive, is necessary if the mathematical formulation is to describe observable natural phenomena. Data in nature cannot possibly be conceived as rigidly fixed; the mere process of measuring them involves small errors. For example, prescribed values for space or time coordinates are always given within certain margins of precision. Therefore, a mathematical problem cannot be considered as realistically corresponding to physical or economic, etc., phenomena unless a variation of the given data in a sufficiently small range lead to an arbitrary small change in the solution. This requirement of "stability" is not only essential for meaningful problems which describe the real processes, but also for approximation methods. Any problem which satisfies our three requirements will be called a properly posed (or well-posed) problem in the sense of Hadamard. Problems that are not well-posed in the sense of Hadamard are termed ill-posed. If the problem is well-posed, then it stands a good chance of solution on a computer using a stable algorithm. If it is not well-posed, it needs to be re-formulated for numerical treatment. Typically this involves including additional assumptions, such as smoothness of solution, etc. [63].

Obviously, if the investigated problem is about bulk cargo, then some of the source data, for example, at least $\quad\left\{a_{i}\right\}_{i=1,(n-1)} \quad$ and $\quad\left\{b_{i}^{k}\right\}_{i=1, \overline{1}(i-1)}^{k=\overline{1, K}}, \quad$ are given approximately. Generally, it makes sense to suppose that costs $\quad i=\overline{1,(n-1)}\left\{c_{i, i+j}^{k}\right\}_{j=1, \overline{1,(n-i)}}^{k=\overline{1, K}} \quad$ are $\quad$ given approximately too and that we know the order of 
approximation of the source data.

It is not difficult to see that one-criterion problems (29)-(31) and (36)-(38) can be rewritten in a more compact form:

$$
\left\{\begin{array}{l}
\min _{z \in Z}\{L(z)\} \\
Z \stackrel{\text { def }}{\equiv}\left\{z \in \mathbb{R}_{+}^{\beta}: A z=u\right. \\
\left.A \in \mathbb{R}^{1}(\alpha \times \beta), u \in \mathbb{R}^{\alpha},(\alpha, \beta) \in \mathbb{N}\right\}
\end{array}\right.
$$

where: in the case if the problem (29)-(31) is being investigated, then the objective function $L(z)$ has the form (29); the matrix $A$ contains the variables held constants in the left side of the system (30); the column-vector $u$ contains constants in the right side of the system (30); $\quad \alpha=(K+1) \cdot(n-1)+2$; $\beta=(3 \cdot K+2) \cdot(n-1)+6$; in the case if the problem (36)-(38) is being investigated, then the objective function $L(z)$ has the form (36); the matrix $A$ contains the variables held constants in the left-hand side of the system (37); the column-vector $u$ contains constants in the right side of the system (37); $\alpha=(K+1) \cdot n ; \quad \beta=(3 \cdot K+1) \cdot n-K+2$.

Let's suppose that instead of the problem (39) with the precisely given data, we have the following approximating problem with given approximately source data:

$$
\left\{\begin{array}{l}
\min _{z \in Z_{\delta}}\{L(z)\} \\
Z_{\delta} \stackrel{\text { def }}{\equiv}\left\{z \in \mathbb{R}_{+}^{\beta}: A_{\delta} z=u_{\delta}\right. \\
\left.\delta \in \mathbb{R}^{1}(\alpha \times \beta), u_{\delta} \in \mathbb{R}^{\alpha},(\alpha, \beta) \in \mathbb{N}\right\}
\end{array}\right.
$$

where in the capacity of the proximity measure between the corresponding elements (vectors, matrixes) is chosen the Euclidean metric:

$$
\left\|A-A_{\delta}\right\| \leq \delta,\left\|u-u_{\delta}\right\| \leq \delta
$$

Remark 7. In the problem (40), (41) for simplicity, the extent of error $\delta$ was set equal for all source data. Actually, the error estimate is determined by the vector $\delta^{\text {actual }} \in \mathbb{R}_{++}^{\alpha \cdot(\beta+1)}$ with appropriate choice of norm. EOR.
Obviously, because of source data precision $\left\{A_{\delta} ; u_{\delta}\right\}$, the request of the system $A z=u$ to be a linearly independent system is an unlawful request, so establishment of this fact is practically impossible. Besides, because of source data precision, the system $A_{\delta} z=u_{\delta}$ appears to be an inconsistent system. In other words, the first requirement for well-posedness in the sense of Hadamard is broken. Further, as it is shown in Ref. [66] during the process of solving a particular practical problem (in this work, a problem of finding optimal quarterly plans on multiproduct manufacturing was investigated) using the simplex method, is possible a situations, when for relatively close (with error less than $1 \%$ ) optimal values of the objective linear function $L(z)$ the amount of items planned to be manufactured in accordance with these optimal plans varies within the range of some hundreds, so the investigated problem appears to be an unstable problem, i.e., the third requirement for well-posedness in the sense of Hadamard is broken. Finally, if we suppose that all source data are given precisely and $Z \neq \varnothing$, then, as the following example shows $[67,68]$, the problem (39) (all the more the approximating problem (40), (41)) can have not only one solution: minimum of the objective function $L(z)=z_{3} \quad$ on the set $Z=\left\{z \in \mathbb{R}_{+}^{3}: z_{1}-z_{2}=0\right\}$ is equal to zero and is reached in any point $z=\left(z_{1}, z_{1}, 0\right), \forall z_{1} \geq 0$, i.e., on the half-line determined by the equation $z_{2}=z_{1}, z_{3}=0$. In other words, the second requirement for well-posedness in the sense of Hadamard is broken, and, therefore, in cases like this, it is required to impose additional condition on the desired solution. In case of the investigated problem being a problem of optimal planning (as the problem investigated in this work is), the additional condition, needed for ensuring of unambiguity, can be formulated in the following way: let a cargo transportation or any other work be performed according to the plan $z^{*}$ and let it be required to change this plan, because of change of the source data. Obviously, new source data correspond to 
new optimal plans. Thus, it is absolutely natural to choose the plan which is the least different from the original plan $z^{*}$, because this criterion of choice will entail the least costs of organizational reconstructions, if they were not considered in the formulation of the problem. As the measure of deviation of the new $z^{\text {new }}$ and the original $z^{*}$ plans can be accepted any positively definite quadric quantic $\sum_{i=1}^{\beta} \sum_{j=1}^{\beta} s_{i, j} \cdot\left(z_{i}^{\text {new }}-z_{i}^{*}\right) \cdot\left(z_{j}^{\text {new }}-z_{j}^{*}\right), \quad$ particularly $\quad$ (for instance, if $s_{i, j}=0$ with $i \neq j$ ), can be accepted any weighted second-order deviation $\sum_{i=1}^{\beta} s_{i} \cdot\left(z_{i}^{\text {new }}-z_{i}^{*}\right)^{2}=\left\|z^{\text {new }}-z^{*}\right\|^{2}$. In other words, it is natural to demand for the plans $z^{\text {new }}$ and $z^{*}$ implementation of the inequality $\left\|z^{\text {new }}-z^{*}\right\|^{2} \leq\left\|z^{(39)}-z^{*}\right\|^{2}$, where vector $z^{(39)} \in \mathbb{R}_{+}^{\beta}$ denotes any solution of Problem (39), i.e., $\forall z^{(39)} \in \operatorname{Arg} \min _{Z} L(z)$. For Problem (39), this vector $z^{\text {new }}$ is called a normal solution with respect to the original vector $z^{*}[63,65-70]$. In future, we will use a shorter name "normal solution" and write it as $z^{\text {normal }}$. Obviously, in case of Problem (39) having an unique solution, it coincides with the normal solution $z^{\text {normal }}$. If Problem (39) has many solutions, then there obligatory exists its normal solution $z^{\text {normal }}:$ it follows from the fact that intersection of a finite number of closed sets is a closed set [71], and the set on which the objective function $L(z)$ reaches its minimum is the intersection of three closed sets $\{z: A z=u\}$, $\left\{z: z_{i} \geq 0, \forall i=\overline{1, \beta}\right\}, \quad\left\{z: L(z)=L^{*}\right\}$, where $L^{*}$ is the lower bound of the values of $L(z)$ on the set $Z$. It is not difficult to see that the normal solution of Problem (39) is determined unambiguously [67]. Indeed, assuming that Problem (39) has two different normal solutions $z^{\text {normal (1) }}$ and $z^{\text {normal (2) }}$, we get that: any point $z=\sigma \cdot z^{\text {normal }(1)}+(1-\sigma) \cdot z^{\text {normal }(2)}, \sigma \in(0,1)$ of the segment $\left[z^{\text {normal (1) }}, z^{\text {normal (2) }}\right]$ satisfies the essential constraints as well as the sign constraints $z \geq 0$; objective function $L(z)$ reaches its maximum at all points of the segment $\left[z^{\text {normal (1) }}, z^{\text {normal (2) }}\right]$; the equality $\left\|z^{*}-z^{\text {normal (1) }}\right\|=\left\|z^{*}-z^{\text {normal (2) }}\right\|$ holds, so it follows that:

$$
\left\langle z^{*}, z^{\text {normal }(1)}-z^{\text {normal }(2)}\right\rangle=\frac{\left\|z^{\text {normal }(1)}\right\|+\left\|z^{\text {normal }(2)}\right\|}{2}
$$

in the bisecting point of the segment $\left[z^{\text {normal }(1)}, z^{\text {normal }(2)}\right]$ we have

$$
\left\|z^{\text {normal }(1)}-z^{*}\right\|>\left\|\frac{z^{\text {normal }(1)}+z^{\text {normal }(2)}}{2}-z^{*}\right\| \text {. }
$$

Subject to $z^{\text {normal }(1)} \neq z^{\text {normal (2) }}$, the inequality contradicts the statement that for Problem (39), the vector (39) $z^{\text {normal (1) }}$ is a normal solution with respect to the original vector $z^{*}$.

Thus, for a normal solution $z^{\text {normal }}$, two first requirements for well-posedness in the sense of Hadamard are satisfied. Summing up the above-mentioned, we can say that Problem (40), (41) with given approximately source data does not let us neither judge whether the solution of Problem (39) with given precisely source data is stable, nor judge whether it is unique even, if Problem (40), (41) has these properties. The exact solution of Problem (40), (41) with given approximately source data, as it was shown above, is inefficient for the investigation of Problem (39) with given precisely source data. From the point of view of available data, as the source data of Problem (39) can serve any source data set $\{A ; u\}$, satisfying the condition (41). We should note that, even if Problem (39) primordially was steady, adding any amount of linearly dependent equation to the essential constraints of Problem (39) makes it an unstable problem, though it stays equivalent to it in the classical sense. Therefore, it is necessary to develop such approach for solving problems of linear programming (particularly, Problems (39) and (40), (41)) for which an assumption about linear independence of the 
essential constraints is not needed. Below we shortly describe a stable method of finding approximate normal solution both for Problems (39) and (40), (41). At first, this method was described in the fundamental article [67].

Let's consider Problem (39), where the source data are given precisely. The point of this stable method of finding approximate normal solution of this problem consists in taking for an approximate solution the following element

$$
\begin{aligned}
& z^{\text {regularized }}=\arg \min _{\left\{z: z_{i} \geq 0 \forall i=1, \bar{\beta}\right\}}\left\{\|A z-u\|^{2}\right.
\end{aligned}
$$

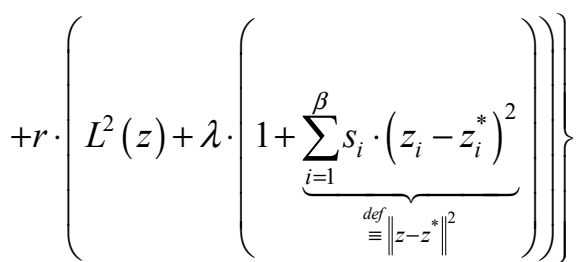

where the parameter $r \in \mathbb{R}_{++}^{1}$ is called a regularization parameter; $\lambda \in \mathbb{R}_{++}^{1} \quad$ is a parameter; vector $z^{*}=\left\{z_{i}^{*}\right\}_{i=\overline{1, \beta}}$ is the fixed original vector, with respect to which the normal solution is searched.

$$
\begin{array}{cccr}
\text { As } & \text { the } & \text { parametric } & \text { functional } \\
M_{\lambda}^{r}[z ; A, u, L] \stackrel{\text { def }}{\equiv}\|A z-u\|^{2}+r \cdot\left(L^{2}(z)+\lambda \cdot\left(1+\left\|z-z^{*}\right\|\right)\right),
\end{array}
$$

called Tikhonov functional, is a quadratic functional and holds $M_{\lambda}^{r}[z ; A, u, L] \stackrel{\|z\| \rightarrow \infty}{\longrightarrow}, \infty$, unique existence of the element $z^{\text {regularized }}$ from Eq. (42) is obvious.

Remark 8. In Eq. (42), the positively definite quadric quantic $\Omega[z] \stackrel{\text { def }}{\equiv} \sum_{i=1}^{\beta} s_{i} \cdot\left(z_{i}-z_{i}^{*}\right)^{2}$ is called a stabilizing functional. If we suppose that choosing criteria (objective functions), not all factors are considered, for example, an absolutely reasonable demand of possible organizational reconstruction being minimal when, due to some reasons, the source data were somehow changed, then the expression $\lambda \cdot\left(1+\sum_{i=1}^{\beta} s_{i} \cdot\left(z_{i}-z_{i}^{*}\right)^{2}\right)$ presented in Eq. (42) can be interpreted as a correction to the influence of neglected factors in the objective function $L^{2}(z)$ proportionally to the magnitude $\lambda \in \mathbb{R}_{++}^{1}$ of expert evaluation. Then, the extent of deviation of the previous criterion (which was chosen without consideration of all factors) from the new criterion can be determined as the least number $\lambda$, such that $\left|L^{\text {old }}(z)-L^{\text {new }}(z)\right| \leq \lambda \cdot(1+\Omega[z])$. Presence of one in the right side of this inequality is necessary, because, if $\Omega\left[z^{*}\right]=0$, then, actually, $L^{\text {old }}\left(z^{*}\right) \neq 0$. EOR.

Substantiation of the described above method of finding approximate normal solution of Problem (39) with given precisely source data follows from the Tikhonov's theorem of normal solution in the case of precise source data [67].

Now we consider Problem (40), (41), where the source data are given approximately. The point of the stable method of finding approximate normal solution consists in taking in the capacity of an approximate solution the

$$
\begin{aligned}
& z^{\text {regularized }}=\arg \min _{\left\{z: z_{i} \geq 0 \forall i=\overline{1, \beta}\right\}}\left\{\left\|A_{\delta} z-u_{\delta}\right\|^{2}\right. \\
& \left.+r \cdot\left(L^{2}(z)+\lambda \cdot(1+\underbrace{\sum_{i=1}^{\beta} s_{i} \cdot\left(z_{i}-z_{i}^{*}\right)^{2}}_{\substack{\operatorname{de}\| \\
=\| z-z^{*} \|^{2}}})\right)\right\}
\end{aligned}
$$

where $\lambda \in \mathbb{R}_{++}^{1}$ is a parameter; vector $z^{*}=\left\{z_{i}^{*}\right\}_{i=\overline{1, \beta}}$ is the fixed original vector with respect to which the normal solution is sought; as the regularization parameter $r \in \mathbb{R}_{++}^{1}$ can be taken a positive root of the equation:

$$
\begin{aligned}
\left\|A_{\delta} z_{r}-u_{\delta}\right\|^{2}= & \delta^{2} \cdot\left(1+L\left(z_{r}\right)+\lambda \cdot\left(1+\left\|z_{r}-z^{*}\right\|^{2}\right)\right)^{2} \\
& -\min _{\left\{z: z_{i} \geq 0 \forall i=\overline{1, \beta}\right\}}\left\|A_{\delta} z-u_{\delta}\right\|^{2}
\end{aligned}
$$

Eq. (44) is called a generalized residual and the method of finding the regularization parameter $r \in \mathbb{R}_{++}^{1}$ as a root of Eq. (44) is called generalized residual principle and, at first, was described in the works $[72,73]$.

Substantiation of the described above method of 
finding approximate normal solution of Problem (39) with given approximately source data follows from the Tikhonov's theorem of normal solution in the case of approximate source data [67].

\section{Conclusions}

In the considered work, there is the mathematical model of optimal planning of multiproduct cargo transportation performed by ships through the given sea route, where every intermediate seaport is simultaneously a departure and destination point and in every of these intermediate seaports are performed handling operations, built. In this work, depending on properties and structure of the feasible solution set, are formulated different optimality conditions; are analyzed different approaches for finding efficient solutions (i.e., Pareto optimal solutions) and for efficiency checking of the obtained solution. The built mathematical model in this work, being a multi-criterion problem of linear programming, using the goal attainment method of Gembicki, is reduced to a scalar problem. Finally in this work is considered and substantiated a stable algorithm (on the basis of Tikhonov regularization) for finding an approximate solution of the derived scalar problem: there is shown applicability of this algorithm in case of given precisely source data as well as in case, when the source data are given approximately.

\section{Acknowledgments}

For the last co-author, the present article was executed within the framework of the State Research Programme "Next Generation Information and Communication Technologies (NexIT)".

\section{References}

[1] Nikolaeva, L. L., and Tsymbal, N. N. 2005. Maritime Transportation. Odessa, Ukraine: FENIX.

[2] Brukhis, G. E., and Luschan, N. A. 1985. Business Operation of Sea Transport. Moscow: TRANSPORT.

[3] James, Jr. B., and Kendall, L. C. 2008. The Business of Shipping. Centreville, USA: Cornell Maritime.

[4] Abusdal, H. 2012. "A Decision Support Methodology for
Strategic Planning Under Uncertainty in Maritime Transportation.” M.Sc. thesis in Marine Systems Design, Trondheim, Norway: Norwegian University of Science and Technology, Department of Marine Technology. (unpublished)

[5] Liotta, G., Stecca, G., and Kaihara, T. 2015. "Optimisation of Freight Flows and Sourcing in Sustainable Production and Transportation Networks." International Journal of Production Economics 164: 351-65.

[6] Kang, M. H., Choi, H. R., Kim, H. S., and Park, B. J. 2012. "Development of a Maritime Transportation Planning Support System for Car Carriers Based on Genetic Algorithm." Applied Intelligence 36 (3): 585-604.

[7] Song, D.-W., and Panayides, Ph. M. 2012. Maritime Logistics: A Complete Guide to Effective Shipping and Port Management. London-Philadelphia-New Delhi: Kogan Page.

[8] Bowersox, D. J., and Closs, D. J. 1996. Logistical Management: The Integrated Supply Chain Process. New York: McGraw-Hill Companies.

[9] Masane-Ose, J. 2014. Competitive position of the Baltic States Ports, Riga, Latvia. KPMG International Cooperative, Transport \& Logistics, 1-12. Accessed January 1, 2017. http://www.rmsforum.lv/ admuploads/file/2_ose_presentation_kpmg_transbaltica_ 2014.pdf.

[10] Masane-Ose, J. 2013. Maritime Cargo Transportation in the Eastern Baltic, Riga, Latvia. KPMG International Cooperative, Transport \& Logistics, 24-7. Accessed January 1, 2017. http://www.digar.ee/arhiiv/et/ perioodika/38391.

[11] Masane-Ose, J. 2013. Competitive Position of the Baltic States Ports, Riga, Latvia. KPMG International Cooperative, Transport \& Logistics, 1-11. Accessed January 1, 2017. http://www.amcham.lv/data/Events/ Competitive\%20Position\%20of\%20the $\% 20$ Baltic\%20Sta tes\%20Ports.pdf.

[12] Medvedeva, A. A. 2014. "Opportunities to Reduce Aggregate Expenditures by Means of Creating a Strategic Alliance by Maritime Cargo Transportation.” M.Sc. thesis, Transport and Telecommunication Institute, Faculty of Transport and Logistics. (unpublished)

[13] Knopp, K. 1954. Theory and Application of Infinite Series. London-Glasgow: Blackie \& Son.

[14] Keisler, H. J. 2010. Elementary Calculus: An Infinitesimal Approach. Stanford, USA: Dover Publications.

[15] Tuy, H., Chinchuluun, A., Pardalos, P. M., Migdalas, A., and Pitsoulis, L. 2008. Pareto Optimality, Game Theory and Equilibria. New York: Springer.

[16] Keeney, R. L., and Raiffa, H. 1993. Decisions with Multiple Objectives: Preferences and Value Tradeoffs. 
Cambridge, USA: Cambridge University Press.

[17] Steuer, R. E. 1986. Multiple Criteria Optimization: Theory, Computation, and Application. New York, USA: John Wiley \& Sons.

[18] Deb, K. 2001. Multi-objective Optimization Using Evolutionary Algorithms. Chichester-New YorkWeinheim-Brisbane-Singapore-Toronto: John Wiley \& Sons.

[19] Saaty, T. L. 1980. The Analytic Hierarchy Process: Planning, Priority Setting, Resource Allocation. New York: McGraw-Hill.

[20] Coello Coello, C. A., and Lamont, G. B. 2004. Applications of Multi-objective Evolutionary Algorithms. Vol. 1: Advances in Natural Computation. New Jersey-London-Singapore-Berlin-Shanghai-Hong KongTaipei-Chennai: World Scientific.

[21] Gearhart, W. B. 1979. "Compromise Solutions and Estimation of the Noninferior Set." Journal of Optimization Theory and Applications 28 (1): 29-47.

[22] Geoffrion, A. M. 1966. "Strictly Concave Parametric Programming. Part I: Basic Theory." Journal of Management Science 13 (3): 244-53.

[23] Geoffrion, A. M. 1966. "Strictly Concave Parametric Programming. Part II: Additional Theory and Computational Considerations." Journal of Management Science 13 (5): 359-70.

[24] Geoffrion, A. M. 1967. "Solving Bi-criterion Mathematical Programs." Journal of Operations Research 15 (1): 39-54.

[25] Geoffrion, A. M. 1968. "Proper Efficiency and the Theory of Vector Maximization." Journal of Mathematical Analysis and Applications 22 (3): 618-30.

[26] Geoffrion, A. M., Dyer, J. S., and Freinberg, A. 1972. "An Interactive Approach for Multi-criterion Optimization, with an Application to the Operation of an Academic Department." Journal of Management Science 19 (4): 357-68.

[27] Balsman, K. L. 1991. "The Theorems of the Alternative." Illinois Wesleyan University, Honors Projects, Paper 3, 29 p. Accessed January 1, 2017. http://digitalcommons.iwu. $\mathrm{edu} /$ cgi/viewcontent.cgi? article $=1000 \&$ context $=$ math_ho nproj.

[28] Giannessi, F. 1984. "Theorems of the Alternative and Optimality Conditions." Journal of Optimization Theory and Applications 42 (3): 331-65.

[29] Giannessi, F. 2001. "Theorems of the Alternative and Optimization." In Encyclopaedia of Optimization, edited by Floudas, Ch. A., and Pardalos, P. M. New York, USA: Springer Publications, 3895-902.

[30] Broyden, C. G. 2001. "On Theorems of Alternative." Journal of Optimization Methods and Software 16 (1-4): 101-11.
[31] Janković, V., Marinković, B., and Raković, S. V. 2013. "Motzkin's Theorem of the Alternative: A Continuous-Time Generalization." Journal of Optimization Letters 7 (8): 1659-68.

[32] Koopmans, T. C. 1951. "Analysis of Production as an Efficient Combination of Activities." In Activity Analysis of Production and Allocation, edited by Koopmans, T.C. New York, USA: John Wiley \& Sons, 33-97.

[33] Von Neumann, J., and Morgenstern, O. 2007. Theory of Games and Economic Behavior. Princeton, USA: Princeton University Press.

[34] Podinovsky, V. V. 1977. "Bi-criterion Problem with Inequivalent Criteria." Transactions of the USSR Academy of Sciences, Series: Technical Cybernetics 5: 44-50.

[35] Podinovsky, V. V., and Nogin, V. D. 2007. Pareto Optimal Solutions of Multicriterion Problems. Moscow: Fiz Mat Lit Press.

[36] Gembicki, F. W. 1973. "Vector Optimization for Control with Performance and Parameter Sensitivity Indices." Ph.D. thesis, Department of System Engineering, Case Western Reserve University, Cleveland, USA.

[37] Gembicki, F. W., and Haimes, Y. Y. 1975. "Approach to Performance and Sensitivity Multiobjective Optimization: The Goal Attainment Method." IEEE Transactions on Automatic Control 29 (6): 769-71.

[38] Fleming, P. J. 1985. "Computer-Aided Control System Design of Regulators using a Multiobjective Optimization." In Proceedings of the Fifth IFAC Workshop on Control Applications of Nonlinear Programming and Optimization, edited by Di Pillo, G., Capri, Italy, June 11-14, 1985, 47-52.

[39] Coello Coello, C. A., Lamont, G. B., and van Veldhuizen, D. A. 2007. Evolutionary Algorithms for Solving Multi-Objective Problems. New York, USA: Springer Science+Business Media.

[40] Fleming, P. J., and Pashkevich, A. P. 1986. "Application of Multiobjective Optimization to Compensator Design for SISO Control Systems." Electronics Letters 22 (5): 258-9.

[41] Hwang, Ch.-L., Md. Masud, A. S. 1979. "Multiple Objective Decision Making-Methods and Applications. A State-of-the-Art Survey." In Lecture Notes in Economics and Mathematical Systems, edited by Beckmann, M., and Kunzi, H. P. Berlin-Heidelberg-New York: Springer-Verlag.

[42] Charnes, A., and Cooper, W. W. 1961. Management Models and Industrial Applications of Linear Programming. New York, USA: John Wiley \& Sons.

[43] Ijiri, Y. 1965. Management Goals and Accounting for Control. Amsterdam, The Netherlands: North-Holland Press.

[44] Lee, S. M. 1972. Goal Programming for Decision Analysis. Philadelphia, USA: Auerbach Publishers. 
[45] Ignizio, J. P. 1976. Goal Programming and Extensions. Lexington, Massachusetts, USA: D.C. Health \& Company.

[46] Ignizio, J. P. 1978. "A Review of Goal Programming: A Tool for Multiobjective Analysis." Journal of the Operational Research Society 29 (11): 1109-19.

[47] Ignizio, J. P. 1985. Introduction to Linear Goal Programming, in: Series: Quantitative Applications in the Social Sciences. Vol. 56. Beverly Hills, USA: SAGE Publications.

[48] Weil, A. 2013. Basic Number Theory. Berlin-Heidelberg: Springer-Verlag.

[49] Davenport, H. 2008. The Higher Arithmetic: An Introduction to the Theory of Numbers. Cambridge, UK: Cambridge University Press.

[50] Eiselt, H. A., and Sandblom, C.-L. 2007. Linear Programming and its Applications. Berlin-Heidelberg-New York: Springer-Verlag.

[51] Karmanov, V. G. 1989. Mathematical Programming. Moscow: Fiz Mat Lit.

[52] Boyd, S., Xiao, L., Mutapcic, A., and Mattingley, J. 2008. Notes on Decomposition Methods. Stanford, USA: Stanford University.

[53] Letocart, L., Nagih, A., and Moungla, N. T. 2012. "Dantzig-Wolfe and Lagrangian Decompositions in Integer Linear Programming." International Journal of Mathematics in Operational Research 4 (3): 247-62.

[54] Kim, K., and Nazareth, J. L. 1991. "The Decomposition Principle and Algorithms for Linear Programming." Linear Algebra and its Applications 152 (1): 119-33.

[55] Ralphs, T. K., and Galati, M. V. 2010. "Decomposition Methods." In Encyclopaedia of Operations Research and Management Science, edited by Cochran, J. J. New York, USA: John Wiley \& Sons.

[56] Dantzig, G. B., and Wolfe, P. 1960. "Decomposition Principle for Linear Programs.” Operations Research 8 (1): 101-11.

[57] Lubin, M., Hall, J. A. J., Petra, C. G., and Anitescu, M. 2013. "Parallel Distributed-Memory Simplex for Large-Scale Stochastic LP Problems." Computational Optimization and Applications: An International Journal 55 (3): 571-96.

[58] Huangfu, Q., and Hall, J. A. J. 2015. Parallelizing the Dual Revised Simplex Method. Edinburgh, Scotland, UK: School of Mathematics, University of Edinburgh.

[59] Hall, J. A. J. 2010. "Towards a Practical Parallelization of the Simplex Method." Computational Management
Science 7 (2): 139-70.

[60] Bixby, R. E., and Martin, A. 2000. "Parallelizing the Dual Simplex Method.” INFORMS Journal on Computing 12 (1): 45-56.

[61] Panyukov, A. V., and Gorbik, V. V. 2012. "Using Massively Parallel Computations for Absolutely Precise Solution of the Linear Programming Problems." Automation and Remote Control 73 (2): 276-90.

[62] Panyukov, A. V. 2010. "Exact and Guaranteed Accuracy Solutions of Linear Programming Problems by Distributed Computer Systems with MPI." Theoretical and Applied Scientific Journal, Series: Natural and Technical Sciences 15 (4): 1392-404.

[63] Tikhonov, A. N., and Arsenin, V. Y. 1977. Solutions of Ill-Posed Problems. New York, USA: Halsted Press.

[64] Courant, R. 1989. Partial Differential Equations. New York-London: Wiley VCH.

[65] Morozov, V. A. 1975. "Linear and Nonlinear Ill-Posed Problems.” Journal of Soviet Mathematics 4 (6): 706-36.

[66] Tikhonov, A. N., Karmanov, V. G., and Rudneva, T. L. 1969. "On the Stability of Linear Programming Problems." In Numerical Methods and Programming, XII. Moscow: Lomonosov Moscow State University Press, 3-9.

[67] Tikhonov, A. N. 1966. "Ill-Posed Optimal Planning Problems." Journal of Computational Mathematics and Mathematical Physics 6 (1): 81-9.

[68] Fedorov, V. V. 1975. "The Instability of Linear Programming Problems." USSR Computational Mathematics and Mathematical Physics 15 (6): 45-58.

[69] Agayan, G. M., Ryutin, A. A., and Tikhonov, A. N. 1984. "The Problem of Linear Programming with Approximate Data." USSR Computational Mathematics and Mathematical Physics 24 (5): 14-9.

[70] Stefanov, S. M. 2001. Separable Programming. Theory and Methods. Dordrecht-Boston-London: Kluwer Academic Publishers.

[71] Kolmogorov, A. N., and Fomin, S. V. 1999. Elements of the Theory of Functions and Functional Analysis. Mineola, USA: Dover Publications.

[72] Goncharsky, A. V., Leonov, A. S., and Yagola, A. G. 1972. "A Certain Regularizing Algorithm for Ill-Posed Problems with an Approximately Given Operator." Computational Mathematics and Mathematical Physics 12 (6): 1592-4.

[73] Goncharsky, A. V., Leonov, A. S., and Yagola, A. G. "A Generalized Residual Principle." Computational Mathematics and Mathematical Physics 13 (2): 294-302. 